\title{
Black phosphorus as a new lubricant
}

\author{
Wei WANG, Guoxin XIE* , Jianbin LUO* \\ State Key Laboratory of Tribology, Tsinghua University, Beijing 100084, China \\ Received: 20 June 2017 / Revised: 18 October 2017 / Accepted: 07 December 2017 \\ (C) The author(s) 2018. This article is published with open access at Springerlink.com
}

\begin{abstract}
In recent years, a new 2D-layered material-black phosphorus (BP) - has been a rising star after the era of graphene owing to its high charge carrier mobility, tunable direct bandgap and unique in-plane anisotropic structure. With the development of the synthesis and modification methods of BP, its extensive applications, e.g., transistors, batteries and optoelectronics have emerged. In order to explore its full potential, research into the tribological properties of BP 2D-layered materials such as lubrication additives and fillers in self-lubricating composite materials would be not only of high scientific value but also of practical significance. In this work, recent advances on the friction and lubrication properties of BP nanosheets made by our group, including the micro-friction properties, the lubrication properties of BP nanosheets as water-based and oil-based lubrication additives, and the friction and wear of BP/PVDF composites will be presented. Finally, the future challenges and opportunities in the use of BP materials as lubricants will be discussed.
\end{abstract}

Keywords: black phosphorus; two-dimensional (2D) material; lubricant additive; self-lubricating composite materials; friction

\section{Introduction}

Friction and wear are primary factors in the energy loss and failure of all types of mechanical systems such as engines [1]. Statistics show that friction consumes a third of the world's primary energy, wear causes approximately $60 \%$ damage of the machine parts and more than $50 \%$ accidents involving machinery equipment originate from lubrication failure and excessive wear [2]. Thus, effective controls and reduction of the friction and wear of moving machineries, can improve the efficiency and lifetime of mechanical parts, and have already become the greatest challenges for today's moving mechanical assemblies.

One of the main purposes of lubrication is to reduce friction and wear. The lubricant medium can be either liquid or solid, or even gas on certain occasions $[3,4]$. Solid lubricants are usually prepared via physical or chemical vapor depositions on the surface of the friction pair. A solid lubricant usually fails within a certain period owing to the finite lubricant thickness, and it can be easily affected by the environment. Liquid lubricant can function efficiently after the formation of a liquid film between the friction pairs [5]. However, larger relative velocity of friction pairs or relatively high viscosity of the liquid is an important prerequisite for the formation of the liquid lubricant film. Antiwear additives and friction modifying additives are frequently blended with lubricants in order to improve the wear resistance and reduce the fuel consumption. In order to minimize wear and friction, various frictionreducing additives are widely used as extreme pressure additives to lubricant oils. The lubrication film formed by tribo-chemical reaction is the key to providing a tool surface with high resistance to seizure. Otherwise, insufficient lubrication and severe wear will occur for example, at the start and stop stages of a machine. It is apparent that the development of new lubricant materials and lubrication methods for achieving high reliability and long service life of machines is still

* Corresponding authors: Jianbin LUO, E-mail: luojb@tsinghua.edu.cn; Guoxin XIE, E-mail: xgx2014@tsinghua.edu.cn 
very essential, owing to the importance of lubrication and the limitations of existing lubrication strategies [6].

Since the first synthesis of graphene in 2004, twodimensional (2D) nanomaterials represented by graphene, molybdenum disulfide $\left(\mathrm{MoS}_{2}\right)$ and hexagonal boron nitride (h-BN) have attracted immense interest in various research areas owing to the combination of remarkable electrical, thermal, optical and mechanical properties and their special atomic structures [7-11]. In recent years, many experimental and theoretical works have been conducted on the mechanical and tribological properties of 2D nanomaterials [12-16]. For instance, many interesting nanotribological properties of 2D materials, e.g., the layer-numberdependent effect of film/substrate binding strength, have been revealed. These works paved the way for the potential application of ultrathin lubricant films in micro/nano-devices. In contrast, the research on the macrotribological properties of these materials has been less extensive owing to the numerous influencing factors. Furthermore, extensive research on these 2D materials as lubricant nano-additives [17-20] and nano-fillers [21-23] in composite materials has been conducted.

Among all the attractive 2D materials, black phosphorus (BP) has recently received considerable attention [24-26]. As a new kind of 2D material, it is still unknown whether it can be potentially used in tribological areas. Based on this consideration, relevant systematic research has been conducted including the synthesis and characterization of BP nanomaterials and their tribological properties, demonstrating that such a kind of $2 \mathrm{D}$ material is promising as a future high-performance lubricant.

\section{Brief introduction of black phosphorus}

Phosphorus belongs to the group $\mathrm{V}$ of the periodic table and has three major allotropes-white phosphorus (WP), red phosphorus (RP), and black phosphorus (BP). BP is nontoxic and the most thermodynamically stable material among all the allotropes of phosphorus at room temperature [27]. BP was first synthesized in 1914, and has aroused research interest as a promising 2D material since 2014. Among the emerging postgraphene 2D-layered nanomaterials, BP is a rising star and has been intensively investigated as the functional material in electronic devices such as fieldeffect transistors owing to its unique properties [28, 29]. A detailed description of the basic characteristics of $\mathrm{BP}$ and its potential applications will be presented in the subsequent sections.

\subsection{Structure of BP}

Similar to other 2D materials such as graphite, BP has a layered structure with the single element $P$, and the layers weakly interact through van der waals forces. However, BP has different structure characteristics as compared to graphite; the phosphorus atom in BP has five valence shell electrons available for bonding, with a valence shell configuration of $3 s^{2} 3 p^{3}$. Each phosphorus atom in layered BP has three bonding nearby atoms through $\mathrm{sp}^{3}$ hybridized orbitals and one lone pair, forming a puckered honeycomb lattice [30]. The schematic diagram of the crystal structure of BP is shown in Fig. 1. The single-layer BP consists of two atomic layers and two kinds of P-P bonds. The bond length between the nearest $\mathrm{P}$ atoms in the same atomic layer is $0.2224 \mathrm{~nm}$, and the bond length between $P$ atoms of different atomic layers is $0.2244 \mathrm{~nm}$ $[31,32]$. Furthermore, there are two inequivalent directions at the edge of BP: zigzag (along the puckered direction) and armchair (perpendicular to the puckered direction). These anisotropic characteristics of BP will be discussed in the later part of this article. The bond angles of the hexagonal structure are configured as $102.09^{\circ}$ and $96.34^{\circ}$ from the top view of BP. The individual layers of BP are assembled by weak van der waals forces and the interlayer distance is $5.3 \AA[33,34]$.
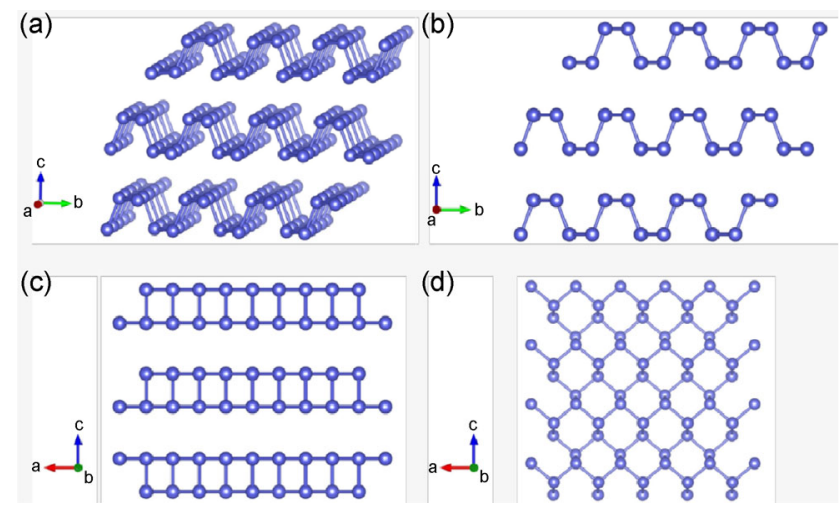

Fig. 1 Schematic diagram of the crystal structure of BP. 


\subsection{Syntheses of BP nanomaterials}

Various kinds of methods have been developed to synthesize BP since Bridgman first successfully converted WP to BP at $200{ }^{\circ} \mathrm{C}$ under 1.2 GPa in 1914 [35]. In contrast to other kinds of allotropes of phosphorus, BP shows good stability at normal temperatures and pressures. Several Japanese groups made important contributions to optimize this method in the 1980s, which significantly improved the quality and size of BP single crystals [36-38]. Phase transformation from $\mathrm{RP}$ to BP has also occurred under the high-energy mechanical milling (HEMM) condition, which also provides high pressures and temperatures. Park et al. reported the synthesis of BP by HEMM using steel balls and milling vessel for $54 \mathrm{~h}$ [39]. Krebs et al. first reported a "low-pressure" synthesis of BP using the catalytic action of $\mathrm{Hg}$ on WP at high temperatures in 1955 [40], and this "low-pressure" method was tremendously promoted by Nilges et al. in 2008 [41]. This low-pressure route method to produce highquality BP included chemical vapor transport reaction method using $\mathrm{Au}, \mathrm{Sn}, \mathrm{RP}$, and $\mathrm{SnI}_{4}$ as reactant sources [27]. Since rediscovery of BP in 2014, it has attracted immense interest, and the synthesis of BP has achieved progress in that the efficiency of synthesis has been greatly enhanced and the quality of BP materials has been improved dramatically.

Similar to graphene and other 2D materials, the preparation methods of phosphorene or few-layer BP have been comprehensively developed. The first method reported in 2014 was micromechanical cleavage followed by transfer onto $\mathrm{SiO}_{2} / \mathrm{Si}$ substrate [42]. As mentioned above, BP is a kind of layered material and the interlayer interaction is dominated by weak van der waals forces. Such a structural feature satisfies the applicable range of the top-down procedure. However, single-layer phosphorus was hard to obtain by the mechanical cleavage owing to the strong binding forces among the layers. Lu et al. reported a method using Ar plasma to thin few-layer BP to monolayer phosphorene [43], and the combination of mechanical cleavage and plasma ablation was promising for controlling the layer number of BP.

The aforementioned methods are effective for producing few- and monolayer $\mathrm{BP}$, but not suitable for mass production. In order to avoid this limitation, liquid phase exfoliation was reported as a reliable method to produce low-size flakes of few- and single-layer BP in greater quantities [44]. The stable dispersions of BP were prepared via ultra-sonication, wherein collapsing cavitation bubbles yielded intense tensile and shear stress fields that exfoliated and fragmented bulk crystals. In order to achieve thinner nano-flakes, aprotic and polar solvents such as $\mathrm{N}$, $\mathrm{N}$-dimethylformamide (DMF), and dimethyl sulphoxide were used to form uniform and stable dispersions. As compared with top-down methods, the bottom-up methods are infrequently used. The chemical synthesis of phosphorene has many advantages such as good crystallinity and high purity. Although chemical bottom-up methods have not made a breakthrough, the rapid development of chemical vapor deposition substrate creates a promising future for phosphorene chemical growth [45]. The requirement for high quality, strong controllability and efficiency will further increase with the development of the field of nanoelectronics.

\subsection{Basic physic-chemical properties of BP}

\subsubsection{Physical properties}

Contrary to graphene, BP is a semiconductor with a thickness-dependent bandgap. As a result of the interlayer interaction, different thicknesses of BP film correspond to different bandgap values ranging from $0.3 \mathrm{eV}$ in the bulk to $2.0 \mathrm{eV}$ in a single layer [46, 47]. It indicates that any particular value of bandgap within the range can be obtained by controlling the thickness. The tunable bandgap of BP supplies the gap between graphene $(0 \mathrm{eV})$ and transition metal chalcogenides (1.0-2.0 eV) [48-50]. Notably, the energy range of BP covers the near-IR (near-infrared) and mid-IR parts of the electromagnetic wave spectrum, suggesting a promising future in the fields of photo catalysis, photovoltaics, thermoelectric and thermal imaging [51, 52].

Another typical characteristic of $\mathrm{BP}$ is the anisotropic properties on the basis of its special crystal structure. Compared with other 2D materials such as Mo- and W-based transition metal dichalcogenides, the anisotropies of BP are much more apparent. The mechanical properties show strong anisotropy along the armchair and zigzag directions. Jiang et al. studied the Young's modulus values of BP via first-principles calculation 
and determined the apparent difference between armchair and zigzag directions [53]. In the case of monolayer phosphorene, the Young's moduli for the armchair and zigzag directions were $21.9 \mathrm{~N} \cdot \mathrm{m}^{-1}$ and $56.3 \mathrm{~N} \cdot \mathrm{m}^{-1}$, respectively, and thus, the value along the zigzag direction was approximately twice that along the armchair direction. Considering phonon dispersion, Qin et al. [54] computed the thermal conductivity of BP. At $300 \mathrm{~K}$, the thermal conductivity along the zigzag direction was $30.15 \mathrm{~W} \cdot \mathrm{m}^{-1} \cdot \mathrm{K}^{-1}$ whereas that along the armchair direction was only $13.65 \mathrm{~W} \cdot \mathrm{m}^{-1} \cdot \mathrm{K}^{-1}$. Using non-equilibrium molecular dynamics, Zhang et al. [55] observed similar anisotropy in that the thermal conductivities of infinitely large monolayer BP along the zigzag and armchair directions were suggested to be $42.553 \mathrm{~W} \cdot \mathrm{m}^{-1} \cdot \mathrm{K}^{-1}$ and $9.891 \mathrm{~W} \cdot \mathrm{m}^{-1} \cdot \mathrm{K}^{-1}$, respectively. They also evaluated the correlation between the thickness and external strain, observed the anisotropic behaviors, and it was determined that the thermal conductivity of BP was layer-independent and the applied strain affected the thermal conductivity in a unique way.

\subsubsection{Chemical properties}

Influenced by the lone pair of phosphorus atoms, BP is very reactive to air, demonstrating limited ambient stability. Light-induced ambient degradation process of BP forms $\mathrm{P}_{x} \mathrm{O}_{y}$ on the surface, which further turns into phosphoric acid. Generally, the degradation of newly exfoliated few-layer BP can be observed after approximately $2 \mathrm{~h}$ under normal conditions. The combined effect of visible light and oxygen $\left(\mathrm{O}_{2}\right)$ contributes to the degradation of $\mathrm{BP}$, and thus, the progress can be hindered by controlling light or $\mathrm{O}_{2}$. A recent study showed that $\mathrm{BP}$ nanoparticles were stable in deaerated aqueous dispersions for weeks [56], suggesting that water interacted weakly with BP. Furthermore, the oxidized surface could effectively prevent the BP underneath from degrading, and therefore, the bulk BP appears to be stable under normal conditions [57].

\subsection{BP applications}

Owing to the aforementioned attractive properties of $\mathrm{BP}$, the exploration of relevant applications has made rapid progress in the fields of electronics, field-effect transistors [58, 59], sensors [60], energy storage [60-62], batteries [63], photocatalysis [64, 65], and optoelectronics [66], and other applications.

\section{Potential of BP as lubricants}

Bulk BP is a layered material in which individual atomic layers are stacked together by van der waals interactions, similar to bulk graphite, $\mathrm{MoS}_{2}$, and h-BN. $\mathrm{BP}$ possesses a unique combination of electronic and optical properties, and thus, it has been intensely investigated for discovering potential applications in electronics and optoelectronics. A solid lubricant is a solid material with low shear strength and high wear resistance. In general, solid lubricants can be used in the forms of powders or coating on the surfaces of contacting pairs, and they can also be dispersed into oils or greases in order to improve the friction and wear performances. Some of the typical features of common solid lubricants are summarized in Table 1.

Few-layer BP which has low shear strength and good thermal stability, is a potential materials using as lubricant additives or composite filler materials. First, BP nanosheets have higher mechanical strength. The ideal elastic moduli of zigzag and armchair BP nanoribbons are approximately 65 and $27 \mathrm{GPa}$, respectively [26]. Morenomoreno et al. [67] investigated the effects of environmental conditions on the mechanical properties of few-layer BP, and the results indicated that the elastic modulus and the breaking strength of BP nanosheets were $46 \pm 10 \mathrm{GPa}$ and $2.1 \pm$ $1 \mathrm{GPa}$, respectively. The in-plane Young's modulus of single-layer BP was $41.3 \mathrm{GPa}$ in the direction perpendicular to the pucker and $106.4 \mathrm{GPa}$ in the parallel direction, and the ideal strains were 0.48 and 0.11 in the perpendicular and parallel directions,

Table 1 The thermal stability and the allowed temperature range of the solid lubricant materialsused in dry tribo-contacts.

\begin{tabular}{cccc}
\hline Materials & $\begin{array}{c}\text { Temperature } \\
\text { range }\left({ }^{\circ} \mathrm{C}\right)\end{array}$ & $\begin{array}{c}\text { Temperature } \\
\text { of thermal } \\
\text { stability }\left({ }^{\circ} \mathrm{C}\right)\end{array}$ & $\begin{array}{c}\text { Coefficient } \\
\text { of friction }\end{array}$ \\
\hline $\mathrm{MoS}_{2}$ & $-184-400$ & 350 & $0.05-0.25$ \\
$\mathrm{WS}_{2}$ & -454 & 425 & $0.05-0.25$ \\
graphite & $-184-650$ & 500 & $0.1-0.3$ \\
PTFE & $-184-260$ & 260 & 0.02 \\
h-BN & $-184-538$ & 700 & $0.1-0.2$ \\
\hline
\end{tabular}


respectively [68]. Second, advancements have been made to enhance the stability of few-layer BP in ambient air $[56,63,64]$. All the properties mentioned above render BP very attractive for achieving low-friction and low-wear regimes. In the following sections, the investigations of the tribological properties of $\mathrm{BP}$ including its friction in the microscale, nanocomposite fillers, and lubrication additives will be discussed.

\section{BP synthesis}

$\mathrm{BP}$, the bulk counterpart of phosphorene, was first synthesized from WP at $200{ }^{\circ} \mathrm{C}$ and under $1.2 \mathrm{GPa}$ in 1914 [35]. In recent years, BP was obtained from WP and RP at high temperatures and high pressures. The synthesis of BP has been widely reported; however, in most of the procedures, extreme operating conditions such as high temperature $\left(>200^{\circ} \mathrm{C}\right)$, high pressure $(>1.2 \mathrm{GPa})$, toxic catalyst were employed.

The layered structure of BP is similar to that of graphite, $\mathrm{MoS}_{2}$ and h-BN. In order to expand the application of BP in the tribological field, the cost of preparation should be considered. The high energy mechanical milling (HEMM) technique at room temperature and normal pressure is a simple method for transforming RP into orthorhombic BP [69]. In the HEMM process, the temperature can rise above $200{ }^{\circ} \mathrm{C}$ and the local pressure can be of the order of $6 \mathrm{GPa}$ in steel vial by the violent collisions of balls. Under these conditions, the transformation from RP to BP is possible. Thus, in this work the HEMM technique was employed for preparing orthorhombic BP.

\subsection{Preparation of BP}

BP powders were prepared using the HEMM technique in a Fritsch P7 premium line planetary ball mill at room temperature and normal pressure. RP powder (purity $>99.999 \%$ ) was purchased from Aladdin Ltd. (Shanghai, China). The materials were weighed initially, and placed in a hardened steel vial $(80 \mathrm{~mL})$ with $10 \mathrm{~mm}$ and $5 \mathrm{~mm}$ milling balls (hardened steel). The weight ratio of $10 \mathrm{~mm}$ ball to $5 \mathrm{~mm}$ ball was 3:1. The weight ratio of milling balls to reagent powders was 30:1. The milling vial was filled with argon gas and set in the ball miller. The milling process was performed for different times. The revolution speed was set to $800 \mathrm{rpm}$. Milling was performed in a sequence of 30 min milling and $10 \mathrm{~min}$ pause. The characterization of the active powder was carried out using powder X-ray diffractometers (XRD; Shimadzu XRD-6000, $\mathrm{CuK} \alpha$ source), scanning electron microscopes (SEM) and transmission electron microscope (TEM, JEM2010HT and JEM-2010FEF). Approximately $10 \mathrm{mg}$ of BP powder was weighed, and subjected to thermogravimetric analysis (Thermo plus EV02 TG8121; Rigaku Corp) in Ar atmosphere with a heating rate of $10{ }^{\circ} \mathrm{C} / \mathrm{min}$. A Raman spectrophotometer (Lab-Ram HR-800; Horiba Jobin Yvon, Inc.) was used to identify the structural units in the BP powders. X-ray photoelectron spectroscopy (XPS) measurement was performed (K-Alpha; Thermo Fisher Scientific Inc.). Monochromatic $\mathrm{Al}-\mathrm{K} \alpha$ radiation $(1486.6 \mathrm{eV})$ was used as the X-ray source. The spectra were calibrated by setting the measured binding energy of the $\mathrm{C} 1$ s peak of adventitious carbon accumulated in the analysis chamber of the spectrometerto $284.7 \mathrm{eV}$. Samples were transferred to the XPS analysis chamber by using a vessel in which the samples were packed in dry Ar gas to avoid structural changes of the sulfides after exposure to air.

\subsection{Results and discussion}

Figure 2(a) shows the XRD results of RP in the XRD image. Initially, the starting RP powder has two broad peaks at $2 \theta=30^{\circ}$ and $55^{\circ}$, suggesting the amorphous structure of RP, which is consistent with previously reported amorphous RP [60, 69, 70]. The TEM electron diffraction pattern shows a diffuse ring of RP (Fig. 3(a)), confirming the amorphous nature of RP. The phase transformation from crystalline RP to BP in the ball milling process depends sensitively on the milling time. After ball milling for only $2 \mathrm{~h}$ (as shown in Fig. 2(a)), the diffraction peaks of RP disappear and instead distinct diffraction peaks appear at $2 \theta=$ 25,35 and $56^{\circ}$, which is consistent with standard orthorhombic BP (JCPDS No. 76-1957), suggesting a phase transformation of RP to orthorhombic BP. The high-resolution TEM (HRTEM) images (Fig. 3(b)) and combining with the XRD analysis results demonstrate the orthorhombic BP in the black powder. 

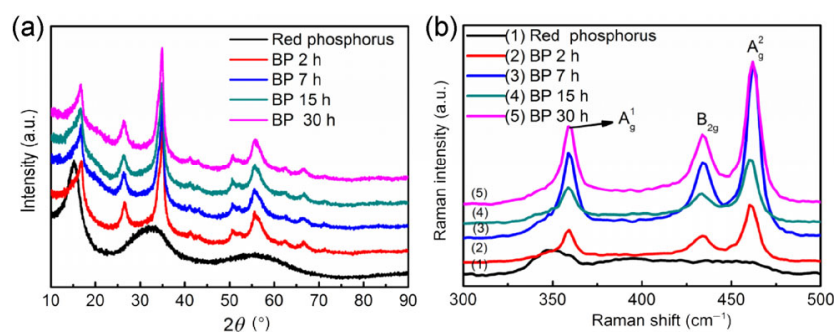

Fig. 2 (a) XRD pattern of RP material withdifferent ball-milling times; (b) Raman pattern of RP material with different ball-milling times.

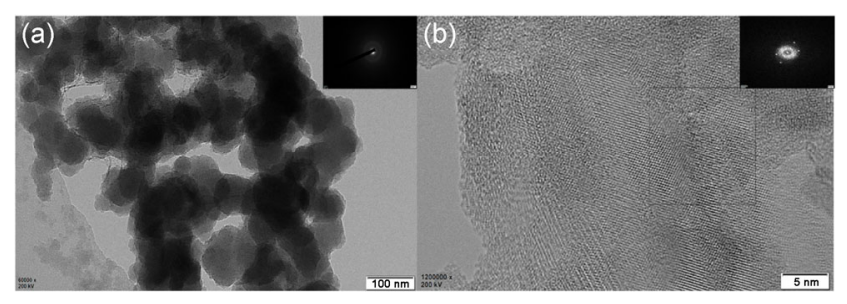

Fig. 3 (a) TEM images of RP; (b) HRTEM images of RP with ball-milling for $2 \mathrm{~h}$.

The XRD patterns and Raman spectra of RP and BP powders at different ball-milling times are also shown in Fig. 2. It can be observed that, as the ball milling time increases, the XRD diffraction peaks become similar. Only the (021) and (111) diffraction peaks widen and shift to a low diffraction angle, indicating that the BP powders become increasingly finer with the increase of the ball-milling time (Fig. 2(a)). Furthermore, the difference in the Raman spectra also demonstrates the transformation of RP into BP powders (Fig. 2(b)). All Raman peaks of BP are much sharper than those of RP, indicating that BP nanoparticles are better crystallized than RP nanoparticles. Futhermore, the broad peak around $348 \mathrm{~cm}^{-1}$ in RP disappears after ball milling, and a new peak at $357.6 \mathrm{~cm}^{-1}$ appears in BP. Three sharp peaks can be attributed to an out-ofplane phonon mode $\left(\mathrm{Ag}_{\mathrm{g}}{ }^{1}\right)$ at $357.6 \mathrm{~cm}^{-1}$, and two in-plane modes $\left(\mathrm{B}_{2 \mathrm{~g}}\right.$ and $\left.\mathrm{A}_{\mathrm{g}}{ }^{2}\right)$ at 431.1 and $459.3 \mathrm{~cm}^{-1}$, respectively $[28,30,39,41]$. A typical SEM image in Fig. 4 shows that the as-prepared BP powder (milling for $30 \mathrm{~h}, 800 \mathrm{r} / \mathrm{min}$ ) is composed of micro particles with a size of approximately $500 \mathrm{~nm}$. The energy dispersive X-ray spectroscopy (EDS) analysis indicates that the content of $\mathrm{P}$ is $91.17 \%$ (wt.\%). The TG curve in Fig. 5(a) shows that the sublimation occurred at approximately $440{ }^{\circ} \mathrm{C}$ in $\mathrm{Ar}$ atmosphere. The resultant BP nanoparticles were further characterized using XPS (Fig. 5(a)). The

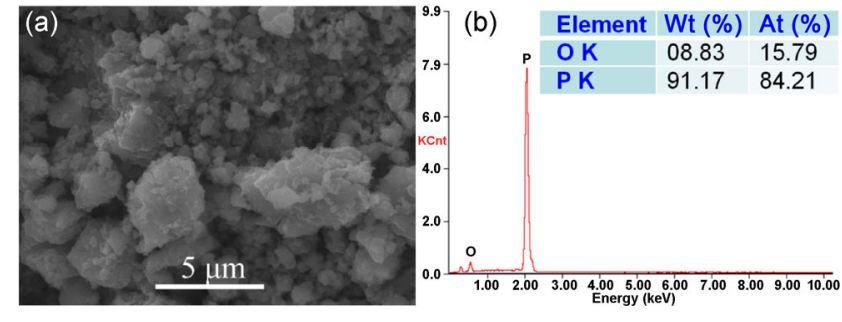

Fig. 4 SEM images of the BP powders at ball-milling for $30 \mathrm{~h}$.
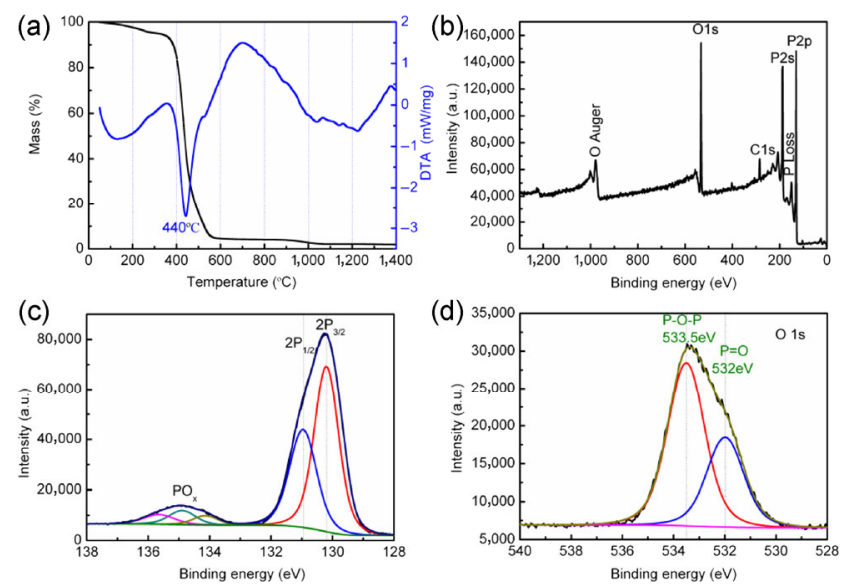

Fig. 5 DTA (a) and XPS (b-d) survey spectrum of black phosphorus powder at BM for $30 \mathrm{~h}$.

absence of other elements apart from $\mathrm{C}, \mathrm{O}$, and $\mathrm{P}$ indicates the high purity of $\mathrm{BP}$ nanoparticles. The two distinct peaks at $129.3 \mathrm{eV}$ and $130.2 \mathrm{eV}$ in the $\mathrm{P} 2 \mathrm{p}$ spectrum are assigned to the $2 \mathrm{p} 3 / 2$ and $2 \mathrm{p} 1 / 2$ orbitals of zero-valent phosphorous $\left(\mathrm{P}^{0}\right)$, respectively. The broad peak at $133.9 \mathrm{eV}$ is attributed to oxidized phosphorus (i.e., $\mathrm{P}^{5+}$ ), indicating the partial oxidization of P0 during sample preparation and purification, as the surface of BP nanostructures is sensitive to oxygen and moisture [70].

\section{BP as a solid lubricant in the microscale}

\subsection{Experimental section}

\subsubsection{Fabrication of BP flakes}

In this section, the influence of oxidation effect on the atomic structure and friction properties of BP will be discussed. The bulk BP was fixed on an iron sheet with adhesion tape. Prior to the experiments, the bulk BP was exfoliated using a Scotch tape to expose the surface of pure BP. Bulk BP (99.99\% purity) was purchased from XFNANO. 


\subsubsection{Influence of oxidation on the atomic structure of BP: Experiment}

A Cypher S (Asylum Research) AFM system was used, and it operated under contact mode to obtain topography and friction data simultaneously. A TR400BP (Asylum Research, AR) cantilever was used for these experiments. The following parameters were employed: the scan rate was $19.53 \mathrm{~Hz}$, integral gain was 65 and setpoint was $1 \mathrm{~V}(0.17 \mathrm{nN})$. The scan sizes of $10 \mathrm{~nm} \times 10 \mathrm{~nm}$ and $20 \mathrm{~nm} \times 20 \mathrm{~nm}$ were used to reduce the data drift and improve the atomic images.

Some of the prepared BP flakes were selected for Raman experiments. The Raman spectra analyses were conducted by using LabRAM HR800 from Horiba. The samples were excited with a $514 \mathrm{~nm}$ green laser, and the lateral resolution was $1 \mu \mathrm{m}$.

\subsubsection{Influence of oxidation effect on the friction properties of BP: Experiment}

A Cypher S (Asylum Research) AFM system was used, and it operated under contact mode to obtain topography and friction data simultaneously. An HQ: XSC11/AIBS cantilever with a spring constant of $0.178 \mathrm{~N} / \mathrm{m}$ and a torsional constant of $660 \mathrm{nN} / \mathrm{V}$, calibrated using an improved wedge method, was used. The setpoint was changed to vary the applied load, and the tip slid on different flakes back and forth with a sliding distance of $100 \mathrm{~nm}$ and a frequency of $4.88 \mathrm{~Hz}$. The friction data was acquired during loading. BP flakes were fabricated on the silicon substrates.

\subsection{Influence of oxidation effect on the atomic structure of BP}

The atomic image of BP obtained using AFM is shown in Fig. 6. The theoretical atomic structure includes two "layers": the upper and lower layers. However, owing to the honeycomb structure, only the phosphorus atoms of the upper layer on the sample surface were scanned. Thus, each unit shown in the figure was observed to have four atoms rather than six ones, confirming the special "puckered" and layered structure of BP.

The Raman spectra of fabricated BP flakes at various air exposure times are shown in Fig. 7. Three prominent peaks in the spectra can be observed at 362,439 and $467 \mathrm{~cm}^{-1}$, corresponding to $\mathrm{A}_{\mathrm{g}}^{1}, \mathrm{~B}_{2 \mathrm{~g}}$ and $\mathrm{A}_{\mathrm{g}}^{2}$,

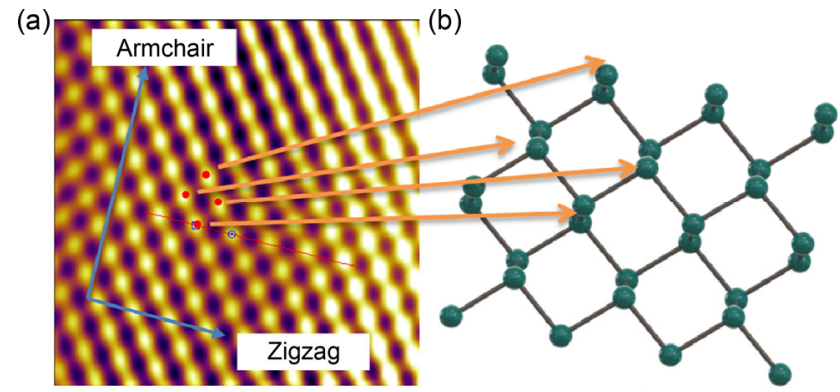

(c)
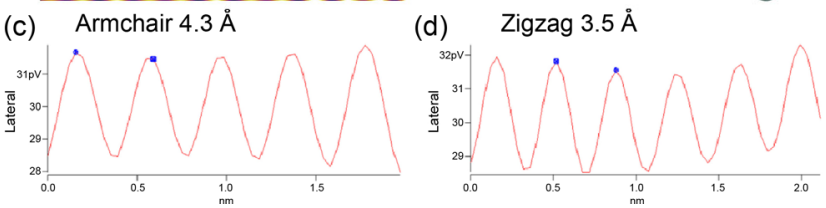

Fig. 6 Corresponding relation between the measured and calculation value: (a) the atomic image of BP; (b) the schematic diagram of BP; (c) and (d) interatomic spacing of armchair and zigzag.

respectively [71]. Figure 7 shows that the intensities of the three peaks depend on air exposure time. The newly-exfoliated BP flake presents the strongest Raman intensities, which are much larger than those of the flakes after exposure to air. A decrease in the peak intensity can be observed over time monotonously, which disappears gradually after $80 \mathrm{~min}$ of exposure in ambient conditions. Specifically, the change in the Raman intensities can be divided into three steps: from 0 to $50 \mathrm{~min}$, the peak intensities decrease gradually with the reduction in the rate of decrease; from 50 to $80 \mathrm{~min}$, the peak intensities show a rise of $30 \%$; after $80 \mathrm{~min}$, the Raman intensities remain steady.

The oxidation reaction under ambient conditions occurs on the surface of BP owing to the existence of oxygen molecules, water molecules in the air and visible light, modifying the properties of the sample. The visible light generated by excitation stimulates charge carriers between oxygen molecules and the BP surface. During the initial period (0-50 min), the volume of the BP sample increases under ambient conditions over time, owing to the aforementioned oxidation process, reducing the Raman intensities of $\mathrm{BP}$ and preventing oxygen molecules from contacting the inner layers of BP, which minimizes the oxidation process. However, long-term exposure to ambient conditions leads to a layer-by-layer etching process; consequently, the swollen sample shrinks and even reduces to the single-layer thickness [72]. Thus, when 

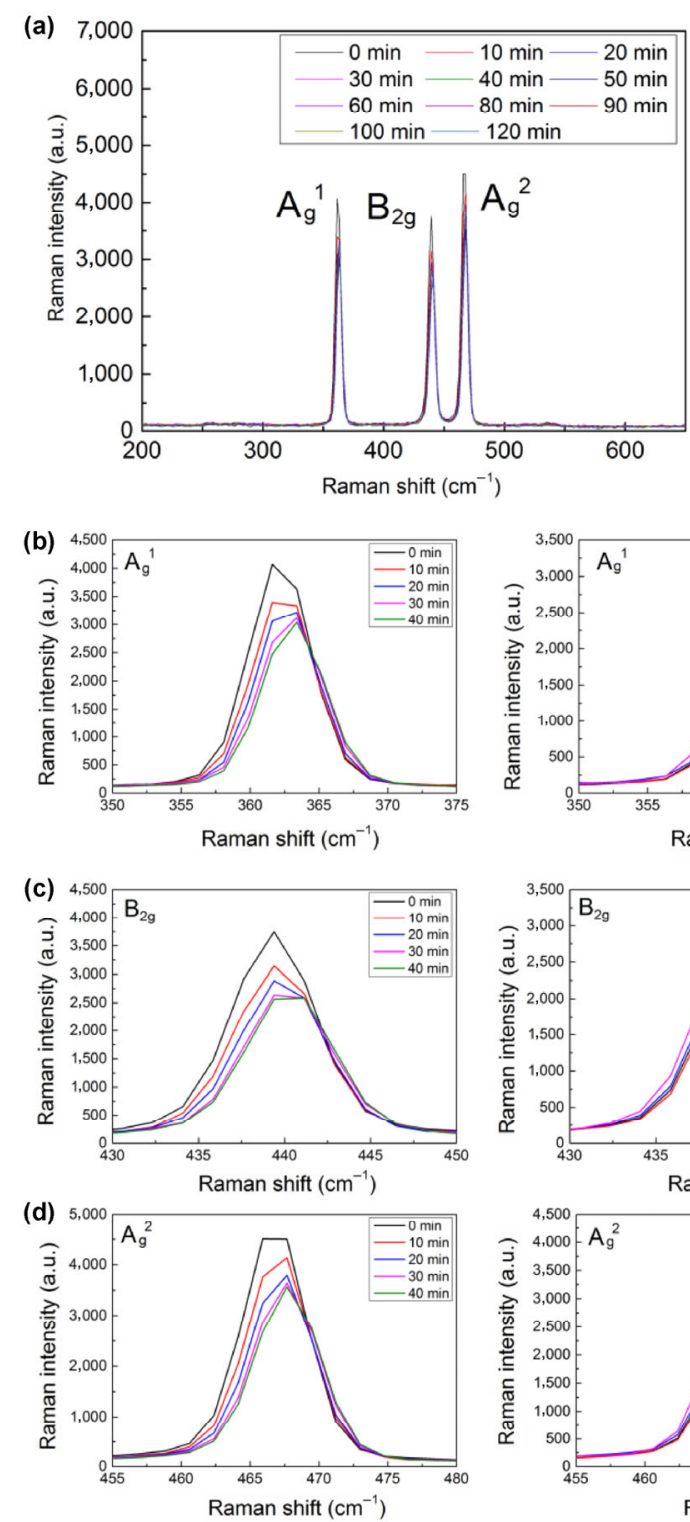
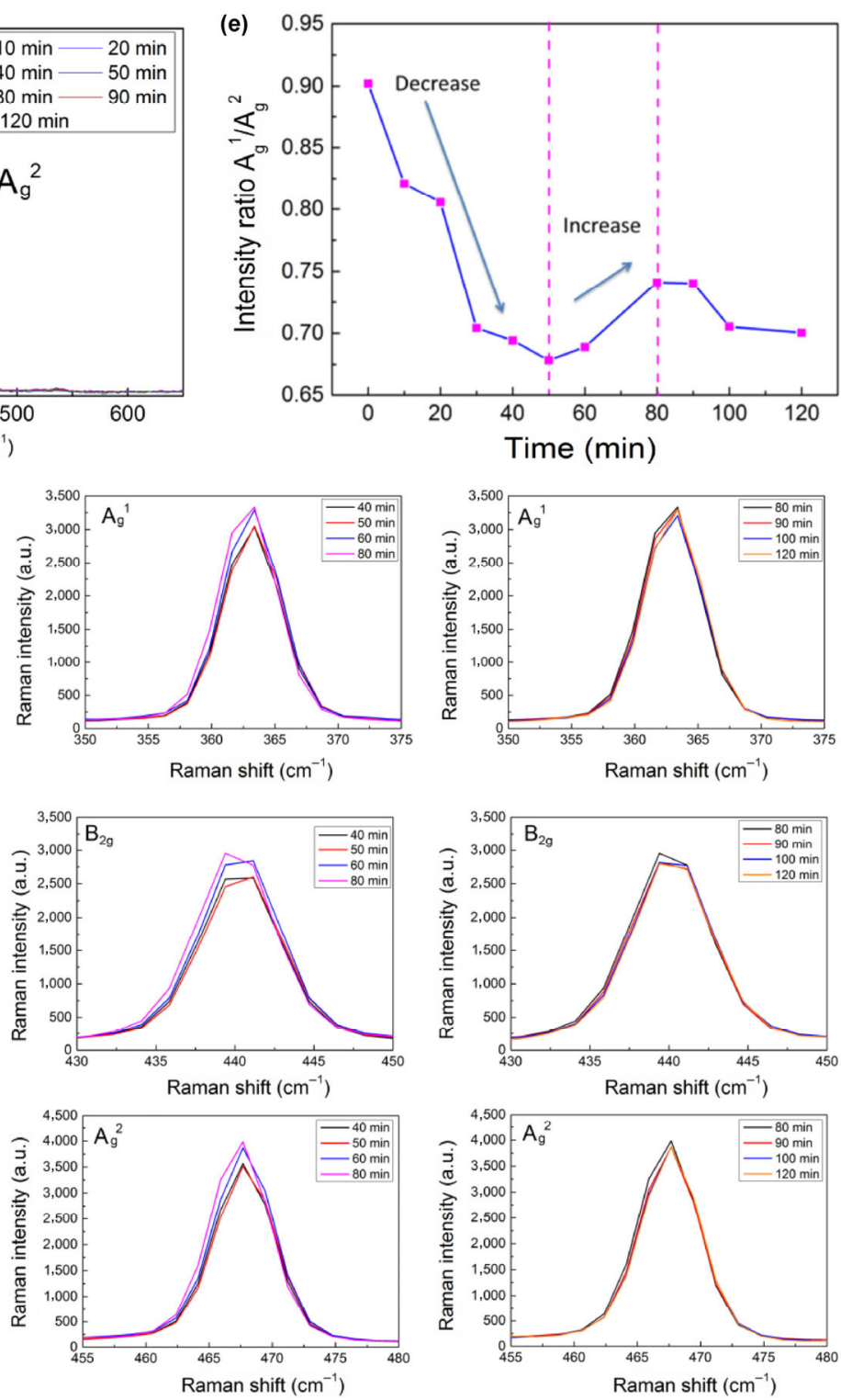

Fig. 7 The Raman characterization result of BP with air exposure time for two hours. (a) Raman spectroscopy of BP at ambient environment and various times; (b), (c) and (d) are the Raman intensities of $A_{g}^{1}, B_{2 g}$ and $A_{g}^{2}$, respectively; (e) The dependence of the intensity ratio between $\mathrm{A}_{\mathrm{g}}^{1}$ and $\mathrm{A}_{\mathrm{g}}^{2}$ on air exposure time.

the oxidation rate slows down and is less than the etching rate (from 50 to $80 \mathrm{~min}$ ), the volume of the oxidation layer on the BP sample surface starts to decrease, and subsequently, the Raman intensity increases. The Raman intensity maintains remains steady after $80 \mathrm{~min}$ of exposure to air, both by oxidation reaction and the etching process.

\subsection{Influence of oxidation effect on the friction properties of BP}

To investigate the effect of oxidation on the friction properties of $\mathrm{BP}$, the friction at various air exposure times were studied. As shown in Fig. 8, the friction of $\mathrm{BP}$ tends to be larger over the measurement period. Specifically, the increase of the friction is not obvious, which is considered to be stable for the initial $30 \mathrm{~min}$ from the newly-fabricated form of BP. However, the friction increases considerably after $30 \mathrm{~min}$ and a friction increase of over $200 \%$ at 120 min has been observed. Initially, sliding occurs between the contact region of fresh BP surface and the AFM tip. Owing to their two-dimensional structure, newly-exfoliated BP 


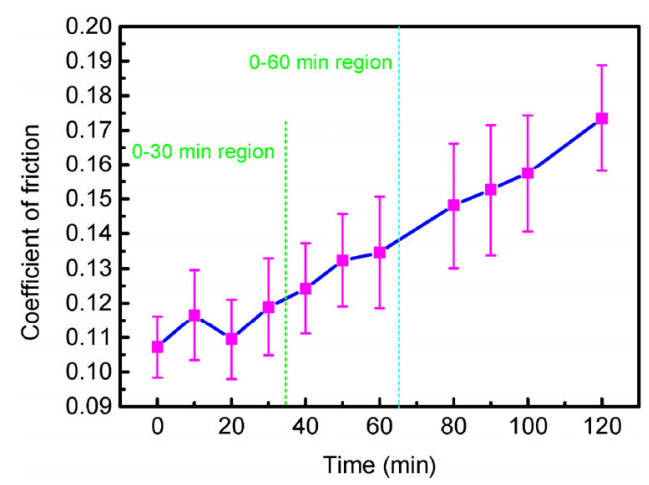

Fig. 8 The dependence of BP friction on air exposure time, under $119 \mathrm{nN}$ normal load (Setpoint=1 V).

samples exhibit relatively low friction. As mentioned above, the pure BP layer tends to react with oxygen molecules, water molecules and visible light, forming an oxide layer that consists of $\mathrm{P}_{2} \mathrm{O}_{5}$ and $\mathrm{p}-\mathrm{P}_{2} \mathrm{O}_{4}[72,73]$. In this case, sliding occurs between the oxide layer and the AFM tip, resulting in the increase of friction.

\section{BP nanosheets as oil-based lubricant additive}

\subsection{Preparation of ultrathin BP nanosheets in water}

In the previous subsection regarding the friction properties of BP in the microscale, the BP nanosheets were prepared via mechanical exfoliation with a Scotch tape. This method has low efficiency and the prepared BP flakes are susceptible to ambient exposure, leading to an irreversible degradation into oxidized phosphorus compounds. In order to harvest the predominant properties of the material, optimized synthesis strategies are required to produce large quantities of exfoliated nanosheets. Liquid exfoliation routes, however, are potentially appropriate for the facile large-scale production of 2D nanomaterials, wherein sonicationinduced cavitation and shear are often used together to separate layers from a solvated bulk material.

At present, the most popular and common way of liquid exfoliation to produce mono- or few-layer BP from bulk BP adopts an organic phase as the solvent. These solvents such as N-methyl-2-pyrrolidone, DMF, dimethyl sulfoxide and $\mathrm{N}$-cyclohexyl-2-pyrrolidone have high boiling points, and are difficult to dismantle in post-processing to aqueous solutions. Herein, we established a scalable, high-yield and environmentally benign method for preparing BP nanosheets via ultrasonication in anhydrous ethyl alcohol.

BP powders were prepared using HEMM technique and stored in an Ar glove box. Anhydrous ethyl alcohol was purged with ultrahigh purity grade Ar for at least $1 \mathrm{~h}$ to remove the dissolved oxygen. Ultrapure water $\left(18.25 \mathrm{M} \Omega \cdot \mathrm{cm}, 25^{\circ} \mathrm{C}\right)$ was used to prepare all the solutions. The BP nanosheets were prepared using a simple liquid exfoliation technique involving ultrasound probe sonication followed by bath sonication of BP powders. Further, $20 \mathrm{mg}$ of BP powder was added to $40 \mathrm{~mL}$ of anhydrous ethyl alcohol in a $50 \mathrm{~mL}$ sealed conical tube and sonicated with a sonic tip for $3 \mathrm{~h}$ at the power of $1,200 \mathrm{~W}$. The ultrasonic frequency was varied from 19 to $25 \mathrm{kHz}$ and the ultrasound probe worked for $3 \mathrm{~s}$ with an interval of $3 \mathrm{~s}$. The dispersion was subsequently sonicated in an ultrasonic bath continuously for another $10 \mathrm{~h}$ at the power of $300 \mathrm{~W}$. The temperature of the sample solution was maintained below $277 \mathrm{~K}$ using an ice bath. The resulting dispersion was centrifuged for $20 \mathrm{~min}$ at 3,000 rpm, and the supernatant containing BP nanosheets was decanted gently. Subsequently, the BP nanosheets solution was centrifuged for $20 \mathrm{~min}$ at $7,000 \mathrm{rpm}$, and the precipitate was repeatedly rinsed with water and re-suspended in the aqueous solution.

Figure 9(a) shows the AFM images of liquidexfoliated BP samples, and they exhibit a triangular structure. From the SEM of liquid-exfoliated BP samples (Fig. 9(b)), it can be observed that the size of BP nanosheets is approximately $350 \pm 13 \mathrm{~nm}$. Such a result is consistent with the size distribution of BP dispersion. The TEM and HRTEM analyses were carried out to investigate the morphology and crystalline quality of the as-synthesized BP nanosheets and nanoparticle samples. Figures 10(a) and 10(b) reveal the morphology of BP nanosheets collected as precipitate

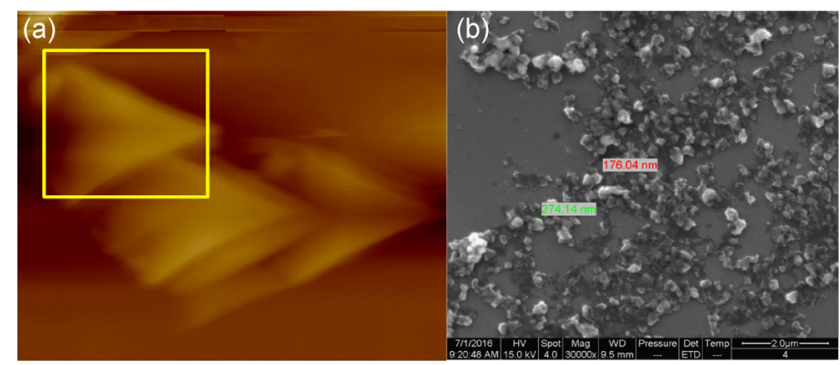

Fig. 9 (a) AFM image and (b) SEM image of BP nanosheets as water based dispersed solution. 


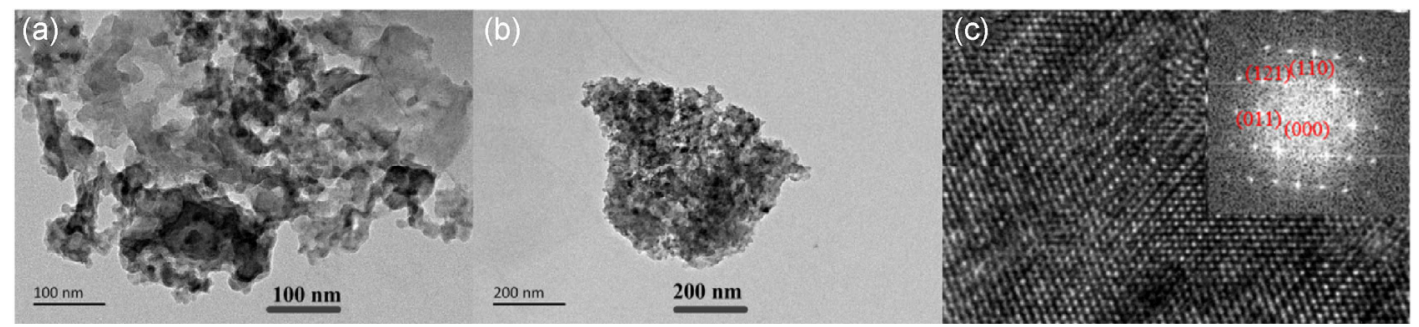

Fig. 10 (a) and (b)TEM images of the black phosphorus nanosheets obtained at 3,000 rpm showing the nanosheets with lengths in few hundreds of nanometers; (c) HRTEM image of the nanosheets.

at 3,000 rpm. The TEM images show that the obtained samples consist of nanosheets with lengths in the range of a few micrometers. Figured 10(c) show the HRTEM images of the same BP nanosheets. The selected area electron diffraction (SAED) pattern, which is consistent with the reported data in the literature [74], confirms that the obtained nanosheets are crystalline in nature and are orthorhombic in crystal structure. The lattice fringe is $0.316 \mathrm{~nm}$, which could be ascribed to the (110) plane of the BP crystal.

\subsection{Preparation and principle of BP nanosheets as oil-dispersible additives}

In this subsection, the synthesis process of the suspensions of BP nanosheets in hexadecane is presented. BP nanosheets were first dispersed in de-ionized water, and this step was completed as described in Section 6.1. The result was a homogeneous dark brown solution of dispersed BP nanosheets. Subsequently, oleylamine was added to the colloidal suspension. Owing to the strong interaction between oleylamine and BP nanosheets, the oleylamine was absorbed on the surface of BP nanosheets, and BP nanosheets became hydrophobic BP nanosheetsoleylamine, floating in water. Subsequently, hexadecane was added to the mixture. The phase transfer process occurred spontaneously, and there was a distinct phase interface between the aqueous phase and hexadecane in 1 day. After removing the aqueous phase using a pipette, the stable suspensions of BP nanosheets in hexadecane were obtained.

\subsection{Lubrication characteristics of BP as oil-dispersible additive at lower loads}

All the lubrication tests were performed using a commercial tribotester (Optimal SRV4) and the mode of the test is reciprocating ball-on-disk. The disk was fixed and the ball was pressed onto the disk, and reciprocating motion was employed with a stroke of $2 \mathrm{~mm}$ and a frequency of $50 \mathrm{~Hz}$. The normal load was increased from $10 \mathrm{~N}$ to $70 \mathrm{~N}$. For each test, a new ball and an un-rubbed position of the disk were used. All the tribological tests were performed at least three times to ensure the repeatability of the experimental results. Both the ball and disk were made of bearing steel (AISI 52100); the diameter of the ball was $10 \mathrm{~mm}$ and the surface roughness ( $\mathrm{Ra}$ ) was approximately $18.5 \mathrm{~nm}$. The surface of the disk was polished and the roughness (Ra) was approximately $20.4 \mathrm{~nm}$.

Further, molybdenum disulfide and graphene oxide (GO) were dispersed in hexadecane in the same way as the dispersion of BP nanosheets. From Fig. 11, it can be observed that the coefficient of friction (COF) of hexadecane and three dispersions present three features: (1) BP-16C has similar tribological properties as GO-16C and $\mathrm{MoS}_{2}-16 \mathrm{C}$; (2) The COFs of the three dispersions decreased as the load was increased from 10 to 70 N; (3) From Fig. 11(d), the COF of hexadecane is apparently different and is higher in the early stages (about $200 \mathrm{~s}$ ). As the load was increased from 10 to $70 \mathrm{~N}$, the maximum Hertzian type pressure also increased from 978 to $1,870 \mathrm{MPa}$. This indicates that the maximum Hertzian pressure that hexadecane could sustain was $1,870 \mathrm{MPa}$. In order to further clarify the difference among hexadecane and the three dispersions, the wear properties are shown in Fig. 12. In general, as the load was increased from 10 to $70 \mathrm{~N}$, the wear scar diameter of the balls increased. The wear scar diameters of the three dispersions were in the range of $175-400 \mu \mathrm{m}$, whereas that of hexadecane was in the range of 525-900 $\mu \mathrm{m}$. The wear scar diameters of hexadecane were three times as large as those of the three dispersions, especially at 70 N. For hexadecane, 

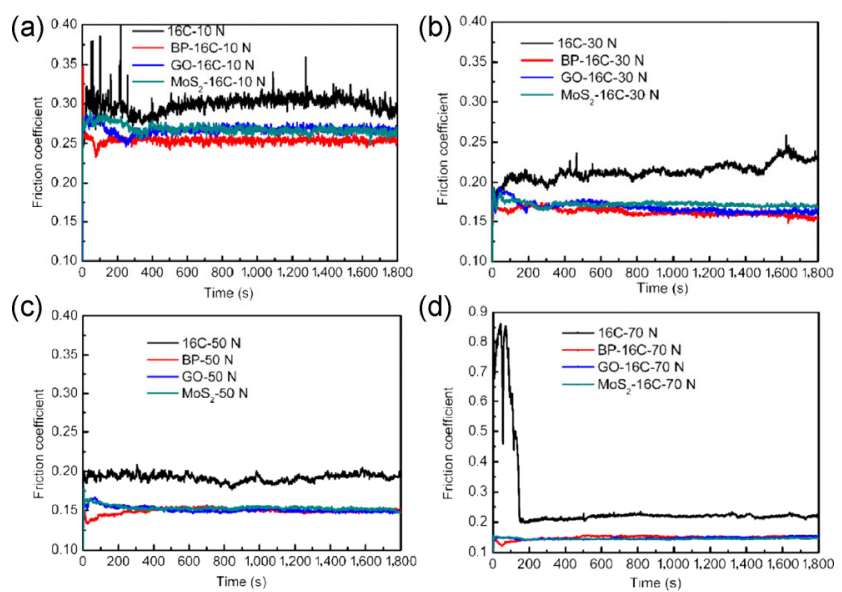

(d)

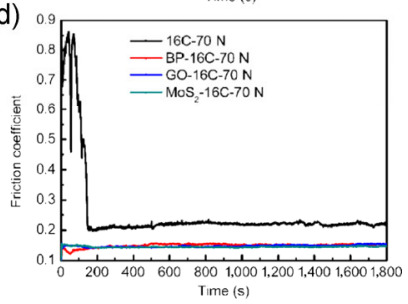

Fig. 11 The coefficient of friction of three dispersion at lower load (16C is the hexadecane, BP-16C: BP nanosheets were dispersed in hexadecane, GO-16C: graphene oxide were dispersed in hexadecane, $\mathrm{MoS}_{2}-16 \mathrm{C}$ : $\mathrm{MoS}_{2}$ nanosheets were dispersed in hexadecane).

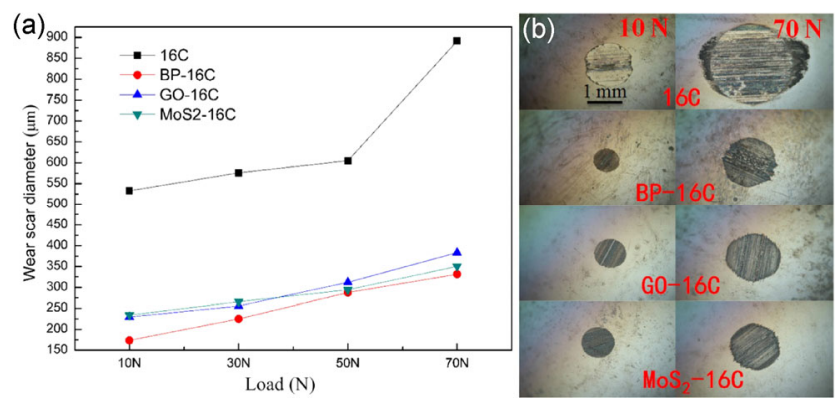

Fig. 12 Wear properties of upper samples (ball): (a) wear scar diameter of balls; (b) OM.

the wear rates significantly improved from 50 to $70 \mathrm{~N}$, as a result of the improvement of COF in this range. For the three dispersions, BP-16C exhibited the smallest wear scar diameters at all the loads, demonstrating the best wear resistance as compared with GO-16C and $\mathrm{MoS}_{2}-16 \mathrm{C}$.

Analyses of the worn surfaces on the lower samples (disk) were performed and the results are shown in Fig. 13. It can be observed from the SEM images that the wear tracks and scars under the lubrication of hexadecane are evident with many scratch lines and deep furrows visible along the sliding direction. In contrast, no evident scratches could be observed on the rubbing tracks under the lubrications of the three dispersions. In the case of worn morphologies, the wear volumes were the largest under the lubrication of hexadecane, whereas those under the lubrications of the other three dispersions were relatively close at $70 \mathrm{~N}$. At lower loads, the wear under the lubrication of BP-16C was minimal. For such phenomena, it is proposed that excess nanosheets accumulating at the inlet of contact area probably interrupt the supply of oil and entry of additive. Overall, it is feasible to choose BP nanosheets as oil-based lubricant additives, and BP nanosheets exhibit comparable lubrication properties with other 2D materials (e.g., GO and $\mathrm{MoS}_{2}$ ).

\subsection{Extreme pressure properties}

In this section, the load-climbing test was used where the normal load was first maintained at $50 \mathrm{~N}$ for $30 \mathrm{~s}$ during the running-in process. Subsequently, the load was increased to $100 \mathrm{~N}$ and maintained for $15 \mathrm{~min}$. Subsequently, the load was increased by $100 \mathrm{~N}$ every 2 min and the test would stop when the COF increased abruptly over 0.3 , indicating that lubrication failure occurred and direct contact (even seizure) between the ball and the disk occurred. The load before lubrication failure was referred to as the highest load without seizure.

In order to investigate the lubrication properties of the four dispersions, the load-climbing test was adopted to fully evaluate the ability of the three kinds of nanosheets to enter the contact area under high loads. $\mathrm{MoS}_{2}-16 \mathrm{C}, \mathrm{GO}-16 \mathrm{C}, \mathrm{BP}-16 \mathrm{C}$ and BP-LP were tested for comparison, and the test temperature was set as $50{ }^{\circ} \mathrm{C}$. BP-LP denotes that BP nanosheets were dispersed in liquid paraffin. As compared with hexadecane, liquid paraffin has higher viscosity. The COF and load over time are displayed in Fig. 14. Notably, the tribological test with BP-LP did not stop until the load reached 2,000 N, which is the highest load that can be provided by the tribotester, which indicates that the highest load with no seizure of the lubricant was no less than 2,000 N. The COF of the lubricant containing BP-16C rose abruptly when the load reached 1,300 N. The highest load of the lubricant containing GO-16C was only $800 \mathrm{~N}$. The performance of the lubricant containing $\mathrm{MoS}_{2}-16 \mathrm{C}$ was the worst and $600 \mathrm{~N}$ was its upper limit of the sustainable load. It is evident that BP-16C has the optimal extreme pressure resistance. 

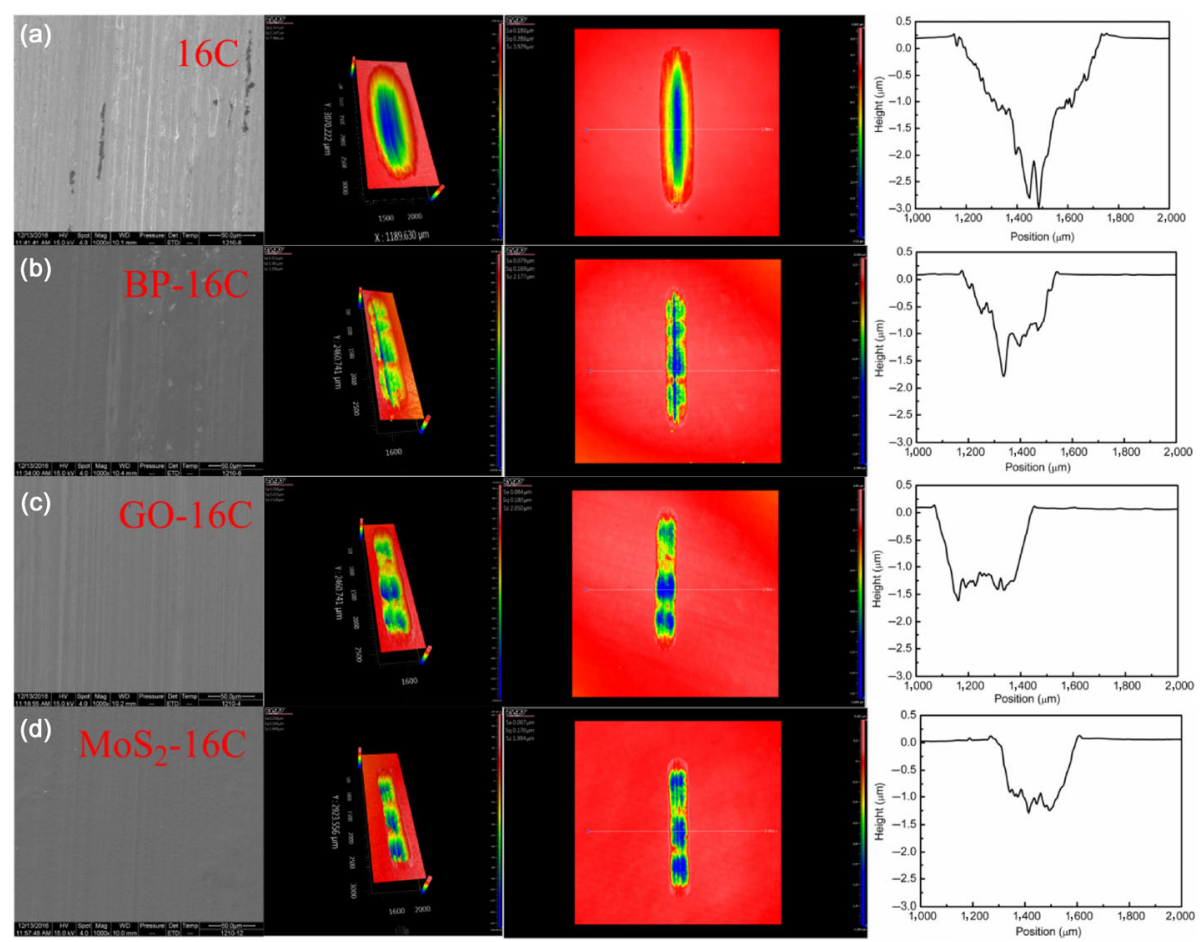

Fig. 13 Wear properties of lower samples (disc) at $70 \mathrm{~N}$ : (a) hexadecane; (b) BP diapered in hexadecane; (c) GO diapered in hexadecane; (d) $\mathrm{MoS}_{2}$ diapered in hexadecane.
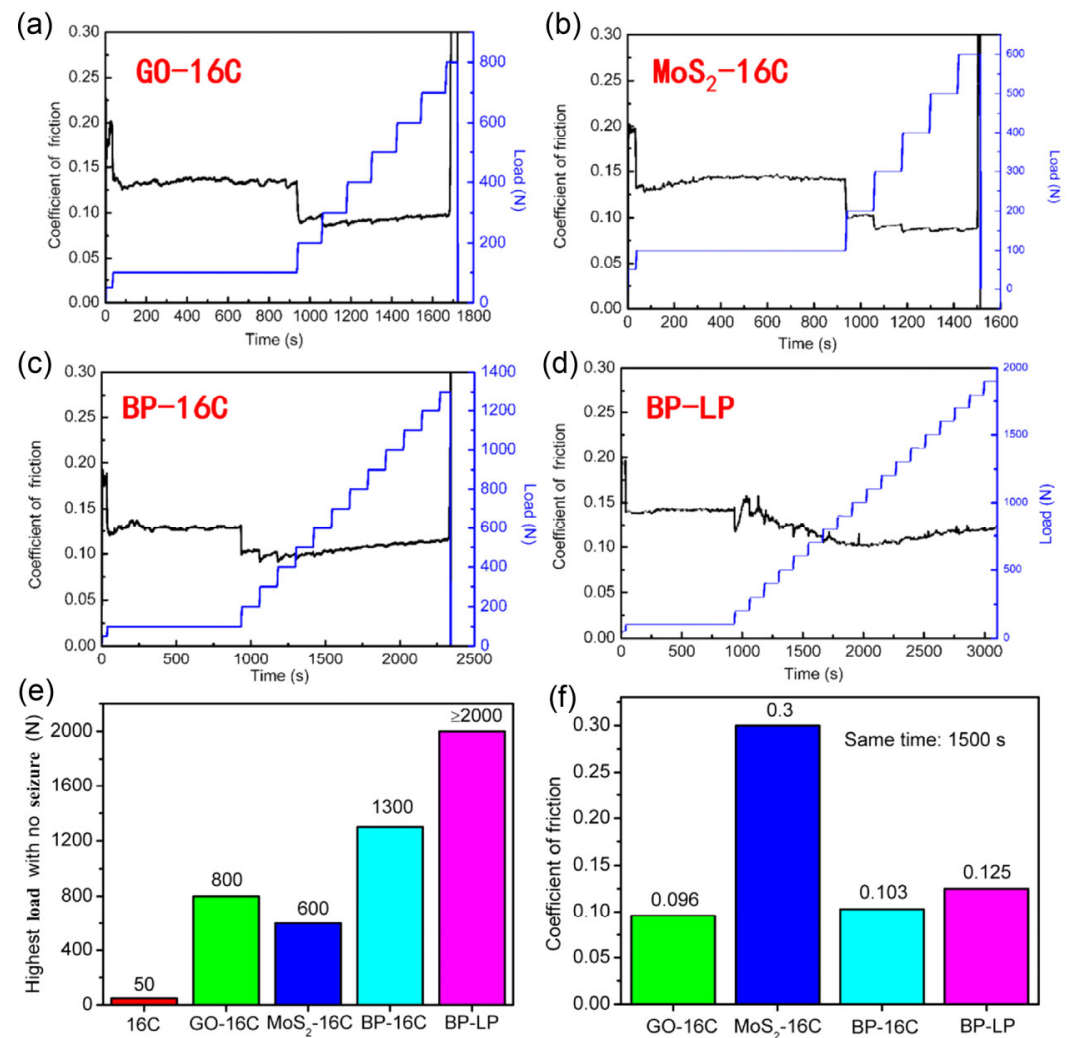

Fig. 14 The results of the load-climbing tribological tests. The tested samples include four lubricants: (a) GO-16C: graphene oxide were dispersed in hexadecane; (b) $\mathrm{MoS}_{2}-16 \mathrm{C}: \mathrm{MoS}_{2}$ nanosheets were dispersed in hexadecane; (c) BP-16C: BP nanosheets were dispersed in hexadecane; (d) BP-LP: BP nanosheets were dispersed in liquid paraffin; (e) Highest load with no seizure of these lubricants; (f) COF of different samples at same time. 


\subsection{Lubrication characteristics of BP as oil-dispersible additives at high loads}

The extreme pressure properties of the three dispersions were evaluated using the load-climbing test, and subsequently, the lubrication properties of the three dispersions were evaluated using SRV4 at different loads for $30 \mathrm{~min}$. Figure 15(a) shows the friction properties of BP-16C at various loads, and it can be observed that, when the load was increased to $400 \mathrm{~N}$ (the corresponding maximum Hertzian type pressure was $3343 \mathrm{MPa}$ ), the COF increased abruptly for the first $60 \mathrm{~s}$ and remained approximately constant thereafter. From Fig. 15(b), it can be observed that the steady COF decreased as the load was increased from 50 to $300 \mathrm{~N}$. The COF increased as the load was increased from 350 to $400 \mathrm{~N}$, and only an incremental rate is less than the decrease rate. From Fig. 15(c), the wear width and wear height also increased as the load was increased, and the wear rate increased abruptly when the load was above $350 \mathrm{~N}$. In order to clarify the reason why the variation trends of friction and wear were different when the load was above $300 \mathrm{~N}$, the surface morphologies and distributions of elements on the worn surfaces are presented in Fig. 16. It can be observed that the surface morphology at $350 \mathrm{~N}$ is different from those below $350 \mathrm{~N}$. In this case, the wear track along the sliding direction is examined. In the case of the element distribution, the concentration of $\mathrm{Fe}$ and $\mathrm{C}$ increased whereas the
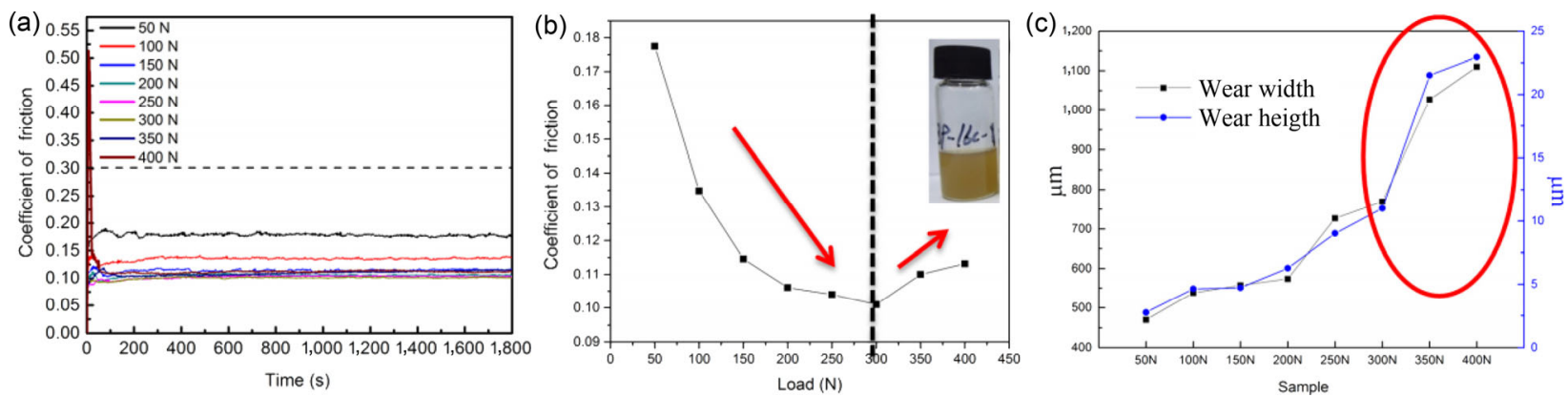

Fig. 15 Friction properties of BP-16C at various loads, (a) COF of BP-16C at various loads; (b) the change trend of steady friction coefficient; (c) wear width and wear height of lower samples (disc).

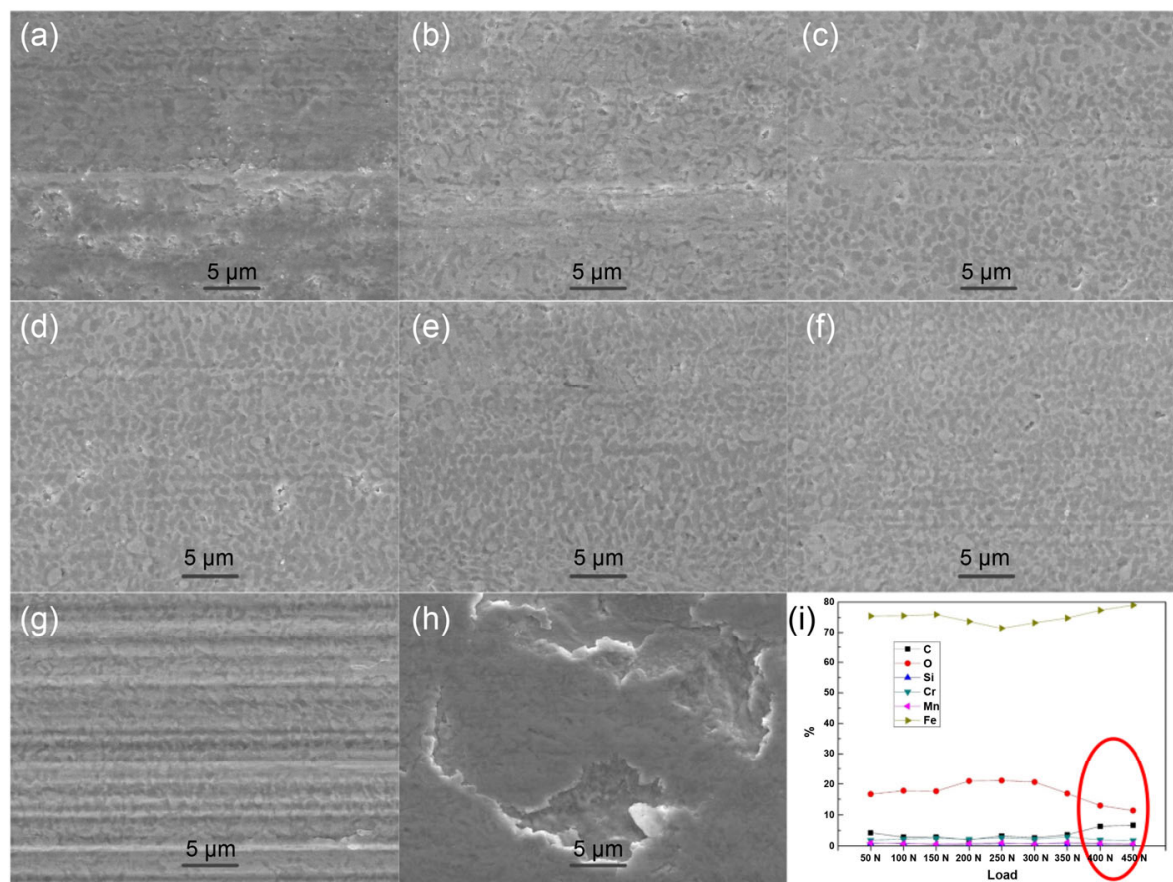

Fig. 16 Surface morphology and distribution of element in worn surface: (a) $50 \mathrm{~N}$; (b) $100 \mathrm{~N}$; (c) $150 \mathrm{~N}$; (d) $200 \mathrm{~N}$; (e) $250 \mathrm{~N}$; (f) $300 \mathrm{~N}$; (g) $350 \mathrm{~N}$; (h) $400 \mathrm{~N}$; (i) distribution of element. 
concentration of $\mathrm{O}$ decreased as the load was increased above $300 \mathrm{~N}$. This suggests that the iron and carbon oxides may be the reason for the decrease in friction. The mechanism of friction will be further discussed in the following section.

The same test methods were adopted for evaluating the lubricating properties of GO-16C. Figure 17 shows the friction properties of GO-16C at various loads, and the steady COF decreased as the load was increased from 50 to $200 \mathrm{~N}$, whereas the COF increased as the load was increased to $250 \mathrm{~N}$ (the corresponding maximum Hertzian type pressure was $2858 \mathrm{MPa}$ ). Two reasons could be proposed for such a phenomenon: on the one hand, the COF increased abruptly for the first $80 \mathrm{~s}$ and remained approximately constant thereafter; on the other hand, when the load reached $250 \mathrm{~N}$, the contact area increased and the Hertzian contact pressure decreased, and subsequently, the steady COF at $250 \mathrm{~N}$ decreased. Figure 17(b) summarizes the wear widths and wear heights of the lower samples (disk); the wear width and wear height also increased as the load was increased, and the wear rate increased abruptly when the load was achieved to $250 \mathrm{~N}$. According to the definition stated previously, the highest sustainable load was $250 \mathrm{~N}$. Figure 18 shows the friction properties of $\mathrm{MoS}_{2}-16 \mathrm{C}$ at various loads. The COF for load above $150 \mathrm{~N}$ increased abruptly for the first $80 \mathrm{~s}$ and remained approximately constant thereafter. The highest sustainable load was $150 \mathrm{~N}$ (the corresponding maximum Hertzian type pressure was 2,411 MPa).

\subsection{Comparison of three oil-based lubrication additives at ultra-low concentration}

It is known that a lubrication additive can enhance the properties of base oil in different aspects. Usually, lubrication additives contain sulfur, phosphorous, etc. However, sulfur compounds in engine exhaust gases are known to poison the catalysts used in exhaust systems to reduce $\mathrm{NO}_{x}$ emissions, and phosphorous is known to poison the hydrocarbon conversion catalysts used in those systems. Simply lowering the
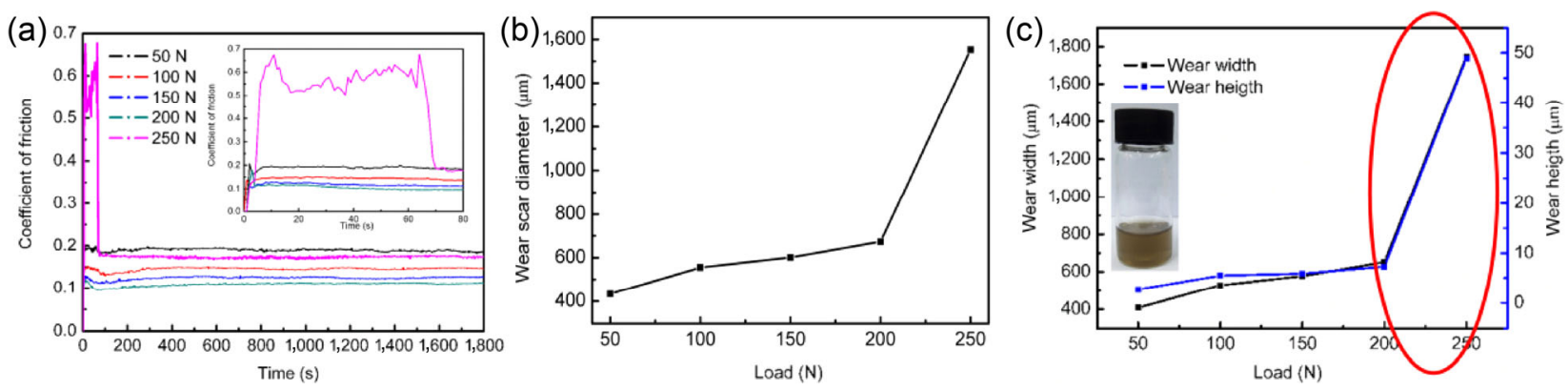

Fig. 17 Friction properties of GO-16C at various loads: (a) COF of GO-16C at various loads; (b) wear width and wear height of lower samples (disc).
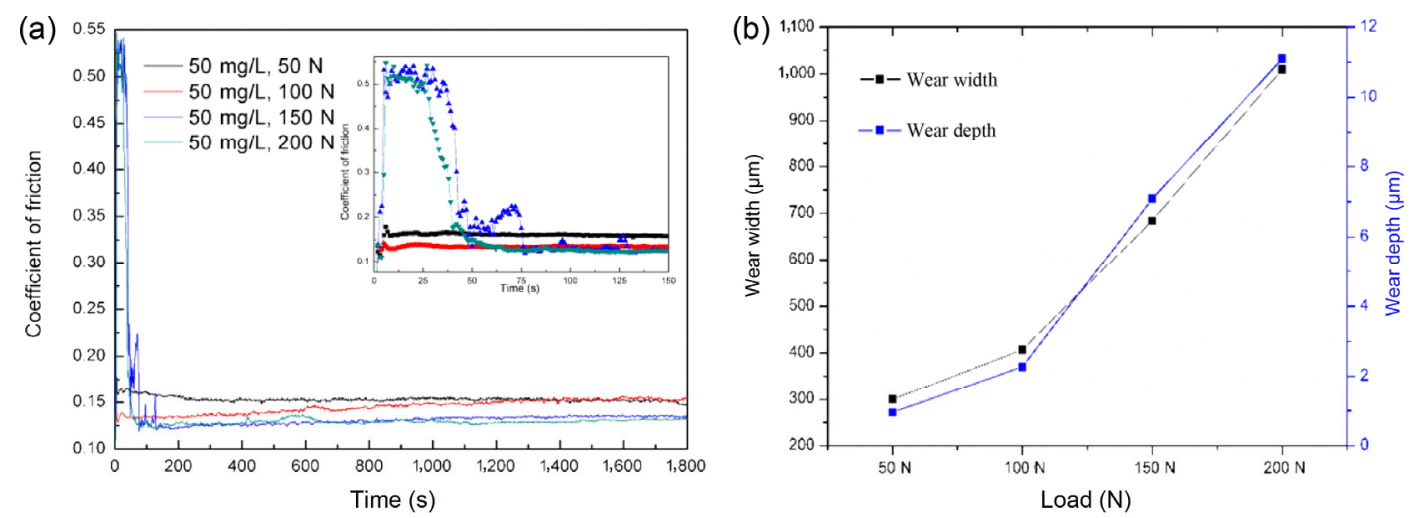

Fig. 18 Friction properties of $\mathrm{MoS}_{2}-16 \mathrm{C}$ at various loads: (a) $\mathrm{COF}$ of $\mathrm{MoS}_{2}-16 \mathrm{C}$ at various loads; (b) wear width and wear height of lower samples (disc). 
amount is not a practical solution to the problem owing to the concomitant reductions of the antiwear properties and oxidation inhibition properties. Thus, the objective is to provide an engine oil for use in conjunction with low sulfur and phosphorous fuels (<350 ppm) for enhanced emission control. Accordingly, the lubrication properties of BP, GO, and $\mathrm{MoS}_{2}$ as additives of ultra-low concentrations will be investigated.

The lubrication properties of the oil dispersions with BP, GO and $\mathrm{MoS}_{2}$ as additives were evaluated using SRV4 at $100 \mathrm{~N}$ for $30 \mathrm{~min}$. The other experimental conditions were the same as the previous part, except that the concentration was varied. Figure 19 is the $\mathrm{COF}$ of three dispersions as additives of various concentrations. The steady COF of BP-16C in Fig. 19(a) decreases as the concentration of the additive concentration was increased from 1 to $10 \mathrm{mg} / \mathrm{L}$. However, when the concentration of BP-16C was decreased to $0.5 \mathrm{mg} / \mathrm{L}$, the COF is unstable and fluctuates between 0.12 and 0.15 . The COF curve of $0.1 \mathrm{mg} / \mathrm{L} \mathrm{BP-16C}$ was different from those with higher concentrations, and the initial COF increased to 0.20 at the first $20 \mathrm{~s}$ and then fluctuated between 0.13 and 0.15 . As the concentration continued to decrease, the COF increased abruptly during the initial period. Thus, on the basis of the definition of the lubrication failure in this work, the lowest sustainable concentration was $0.1 \mathrm{mg} / \mathrm{L}$ (0.1 ppm). Few layered graphene and $\mathrm{MoS}_{2}$ are also the layered materials which stacked along the c-axis by van der waals forces. In recent years, extensive research about the graphene oxide and $\mathrm{MoS}_{2}$ as lubricant additive has been conducted. To further evaluate the comprehensive performances of BP as oil based lubricant additive, the compare study of graphene oxide and $\mathrm{MoS}_{2}$ at ultra-low concentration as oil based lubricant additive are introduced. The COFs of GO-16C at various concentrations were shown in Fig. 19(b). When the concentration decreased to $5 \mathrm{mg} / \mathrm{L}$, the COF is instability and the initial COF increased to 0.6 at the first twenty seconds and then tended to be stable. The COF at $1 \mathrm{mg} / \mathrm{L}$ is entirely different from that of the higher concentration. The initial COF increased to 0.70 at the first twenty seconds and then fluctuated between 0.70 and 0.18 until $280 \mathrm{~s}$ to the steady state. In general, in the low concentration $(<25 \mathrm{mg} / \mathrm{L})$ as the concentration increased the steady state COF decreased. Thus, the ultra-low concentration of GO lubricant additives is different from that of BP. In same situation, the lowest bearing concentration of GO lubricant additives is $5 \mathrm{mg} / \mathrm{L}$ (5 ppm). The COFs of $\mathrm{MoS}_{2}-16 \mathrm{C}$ at various
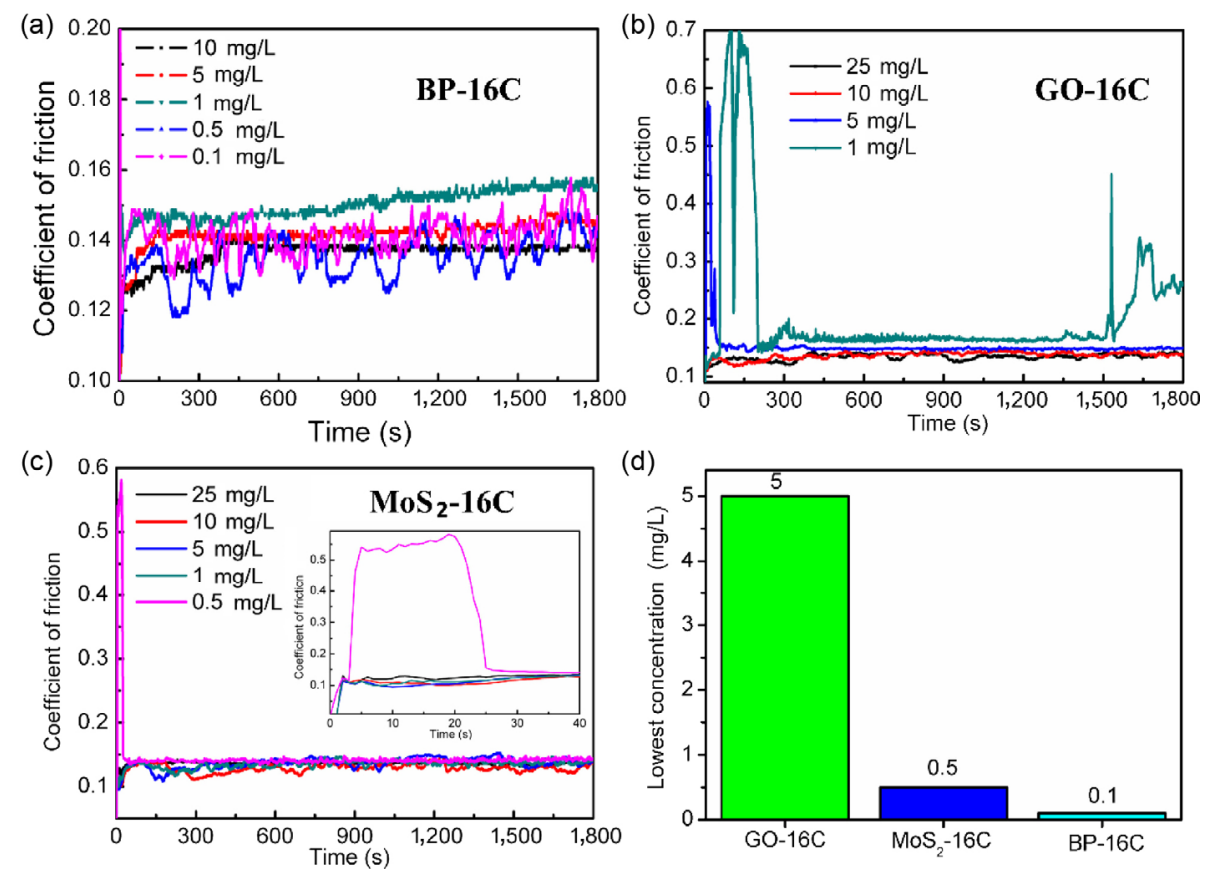

Fig. 19 Coefficients of friction (COF) of BP-16C at various concentrations: (a) COF of BP-16C below $10 \mathrm{mg} / \mathrm{L}$; (b) COF of GO-16C below $10 \mathrm{mg} / \mathrm{L}$; (c) $\mathrm{COF}$ of $\mathrm{MoS}_{2}-16 \mathrm{C}$ below $10 \mathrm{mg} / \mathrm{L}$; (d) Comparison of three oil-based lubrication additives at ultra-low concentration. 
concentrations were shown in Fig. 19(c). The similar method was used for evaluating the ultra-low concentration of $\mathrm{MoS}_{2}-16 \mathrm{C}$. In the concentration from 1 to $25 \mathrm{mg} / \mathrm{L}$, the variety regulation of COF was not obvious. However, when the concentration decreased to $0.5 \mathrm{mg} / \mathrm{L}$, the COF was displayed strikingly different behavior. At this concentration, the COF was instability and the initial COF was increased to 0.6 at the first twenty seconds and then tended to be stability. Thus, the lowest bearing concentration of $\mathrm{MoS}_{2}$ lubricant additives is $0.5 \mathrm{mg} / \mathrm{L}(0.5 \mathrm{ppm})$. Comparison with three oil-based lubrication additives at ultra-low concentration (Fig. 19(d)), BP-16C has best lubrication properties at lowest concentration, 50 times more lubrication properties than that of $\mathrm{GO}-16 \mathrm{C}$, and 5 times more lubrication properties than that of $\mathrm{MoS}_{2}-16 \mathrm{C}$.

\subsection{Comparison analysis of three 2D material lubrication additives at $1 \mathrm{mg} / \mathrm{L}$}

The tests in this section were also performed using a commercial tribotester (Optimal SRV4), and the mode of the test was reciprocating ball-on-disk. The disk was fixed and the ball was pressed onto the disk, and the reciprocating motion was employed with a stroke of $2 \mathrm{~mm}$ and a frequency of $50 \mathrm{~Hz}$ at a temperature of $50{ }^{\circ} \mathrm{C}$. The normal load was $100 \mathrm{~N}$ (the corresponding maximum Hertzian type pressure was 2,106 MPa). For each test, a new ball and an un-rubbed position of the disk were used. All the tribological tests were performed at least three times to ensure the repeatability of the experimental results. Both the ball and disk were made of bearing steel (AISI 52100); the diameter of the ball was $10 \mathrm{~mm}$ and the surface roughness ( $\mathrm{Ra}$ ) was approximately $18.5 \mathrm{~nm}$. The surface of the disk was polished and the roughness ( $\mathrm{Ra}$ ) was approximately $20.4 \mathrm{~nm}$.

In the previous section, the lubricating properties of three 2D materials-BP, $\mathrm{MoS}_{2}$ and GOas oil-based lubrication additives at ultralow concentration were investigated. The results showed that, under these conditions $\left(100 \mathrm{~N}, 50 \mathrm{~Hz}, 50{ }^{\circ} \mathrm{C}\right)$, the lowest bearing concentrations of BP-16C, GO-16C and $\mathrm{MoS}_{2}-16 \mathrm{C}$ were $0.1 \mathrm{mg} / \mathrm{L}(0.1 \mathrm{ppm}), 5 \mathrm{mg} / \mathrm{L}(5 \mathrm{ppm})$ and $0.5 \mathrm{mg} / \mathrm{L}$ $(0.5 \mathrm{ppm})$, respectively. However, there is no direct comparative study among $\mathrm{BP}, \mathrm{GO}$ and $\mathrm{MoS}_{2}$ as oil-based lubricating additives at the same concentration.
In order to further explain the difference among the three 2D materials as lubricant additives at the same concentration, the friction and wear tests were conducted at $1 \mathrm{mg} / \mathrm{L}$. Figure 20 shows the COF of the three $2 \mathrm{D}$ materials as lubrication additives. It can be observed that the COF of hexadecane increased to 0.8 in the first $160 \mathrm{~s}$, and thereafter entered the steady COF (0.18), whereas the COF of the GO at the initial phase was 0.14 and lasted for $55 \mathrm{~s}$, suddenly increased to 0.7 and lasted for $145 \mathrm{~s}$, and finally entered the steady COF (0.167). However, the COFs of BP-16C and $\mathrm{MoS}_{2}-16 \mathrm{C}$ were basically stable and remained at 0.140 . This result indicates that BP-16C and $\mathrm{MoS}_{2}-16 \mathrm{C}$ have similar properties of wear resistance.

\subsection{Lubrication mechanism of BP-16C}

In order to clarify the reason why the oil dispersion with BP-16C as the additive of ultra-low concentrations exhibits good lubrication abilities, the worn surfaces of the upper samples (balls) were analyzed by SEM and EDS (Fig. 21(a)). The chemical compositions of the friction surface mainly contained C, O, P, Cr and Fe. $C$ originated mainly from the base oil (hexadecane), $\mathrm{P}$ originated from the BP nanosheet, and $\mathrm{Cr}$ and $\mathrm{Fe}$ mainly originated from the substrate. The content of $\mathrm{BP}$ in the black wear debris after frictional reaction was $1.51 \mathrm{wt}$ \%, whereas less BP was observed in other regions of shallow color. Thus, BP was rich in wear debris on the surface of frictional balls, and the reaction film on the frictional surface mainly contained $C, F e$ and ferrous oxide. Figure 21(b) shows the sampling place of the worn surface using focused ion beam (FIB), and a Cr film and Pt layer were deposited on the sample surface before FIB cutting to protect

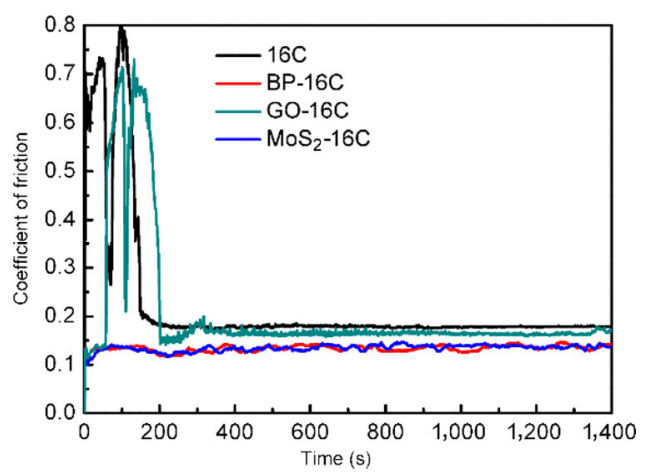

Fig. 20 Compare of coefficients of friction (COF) of three 2D materials lubrication additive at $1 \mathrm{mg} / \mathrm{L}$. 


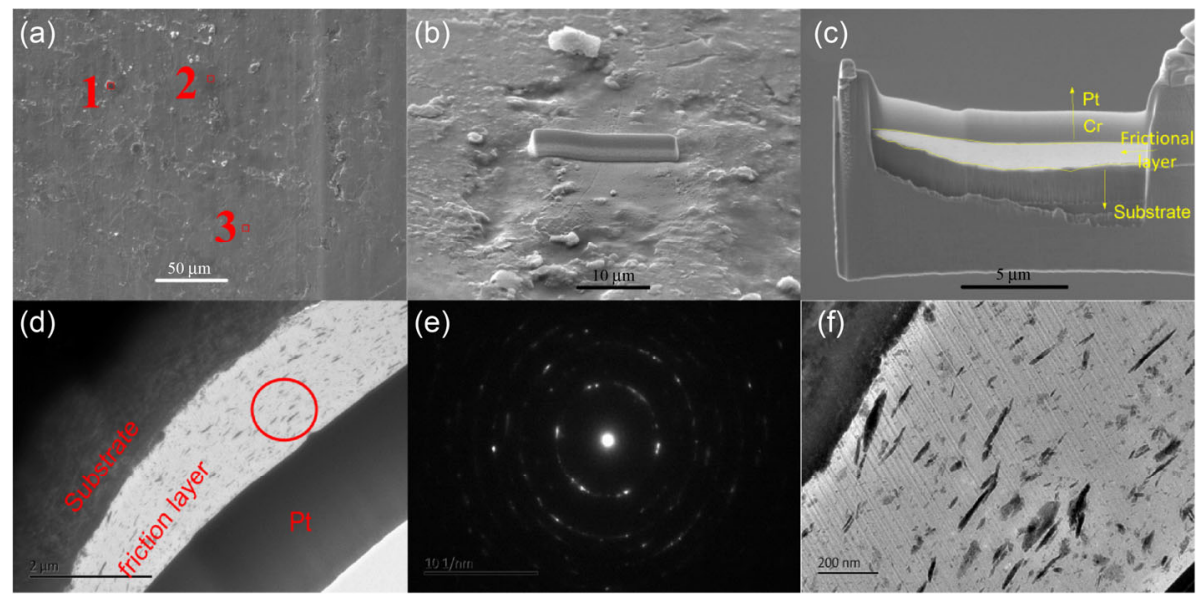

Fig. 21 SEM and TEM images of worn surfaces on ball lubricated with BP-16C at $75 \mathrm{mg} / \mathrm{L}$ : (a) SEM images of worn surfaces on ball; (b) the sampling place of samples using FIB; (c) TEM specimens by preparing in FIB; (d) TEM images of worn surfaces; (e) the selected area electron diffraction (SAED) images in the circle of (d); (f) TEM images of frictional layer.

the tribofilm. Figure 21(c) shows the TEM result of the specimen after fabricating using FIB. It was composed of a protective coating, frictional layer and substrate. The frictional layer had a polycrystalline structure (Figs. 21(d) and 21(e)), and it was mainly composed of black nanosheet, tiny wear debris and white substrate. In order to further explain the chemical compositions of the frictional layer, the distribution of the chemical elements in the frictional layer on the worn surfaces of balls is presented in Fig. 22. The white part in the frictional layer consists largely of $\mathrm{C}$, and the black nanosheets are composed of iron oxide. The content of phosphorus is very difficult to detect in this situation. Thus, the frictional layer on the upper samples (balls) mainly consists of $\mathrm{C}, \mathrm{Fe}$ and ferrous oxide.

The SEM and TEM images of the worn surfaces on the disks lubricated with $75 \mathrm{mg} / \mathrm{L} \mathrm{BP}-16 \mathrm{C}$ are shown in Fig. 23. Local enrichment of debris in the

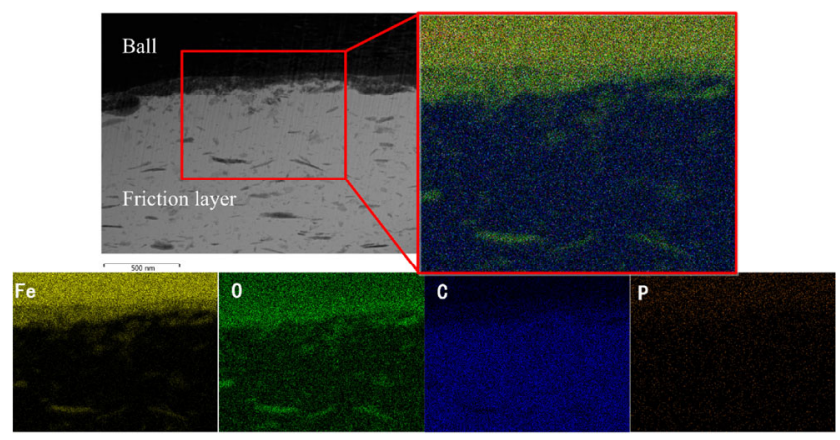

Fig. 22 The distribution of the chemical elements in frictional layer at worn surfaces of balls. wear track can be observed, and the thin nanosheets containing 3.09 wt.\% phosphorus have higher content of phosphorus. The elements $\mathrm{Si}, \mathrm{Mn}$, and $\mathrm{Cr}$ originated from the substrate. Figure 23(b) shows the sampling place of the wear surface using FIB. A Cr layer was first deposited on the sample surface, and subsequently, a $\mathrm{SiO}_{2}$ layer was deposited on the sample surface before the FIB fabrication to protect the tribofilm. Thus, the effect of impurity element $\mathrm{Pt}$ on $\mathrm{P}$ can be significantly alleviated because their characteristic peaks in EDS are close. Figure 23(c) shows the TEM result of the specimen prepared using FIB. It can be observed from Fig. 23(d) that the sample is composed of the $\mathrm{SiO}_{2}$ layer, the friction layer and the substrate. The frictional layer is in homogeneously distributed, and the layer thickness was 20-200 nm. Dark and white areas were present in the frictional layer, whereas the chemical composition will be discussed in the following section. After the frictional reactions, the substrate exhibited various characteristics. Large plastic deformation was observed in the zone near the frictional layer, and the nanocrystal line was present in the red circle 4 of Fig. 23(d). From the surface of the frictional film to its interior, the microstructures transformed from a nanocrystal line to single crystals. During the friction process, there was plastic displacement of the metal on the substrate surface and the ball surface. Severe plastic deformation on the surface can improve the surface hardness, and thus, it is helpful to improve the wear resistance (Fig. 23(f)). Figure 24 shows the 


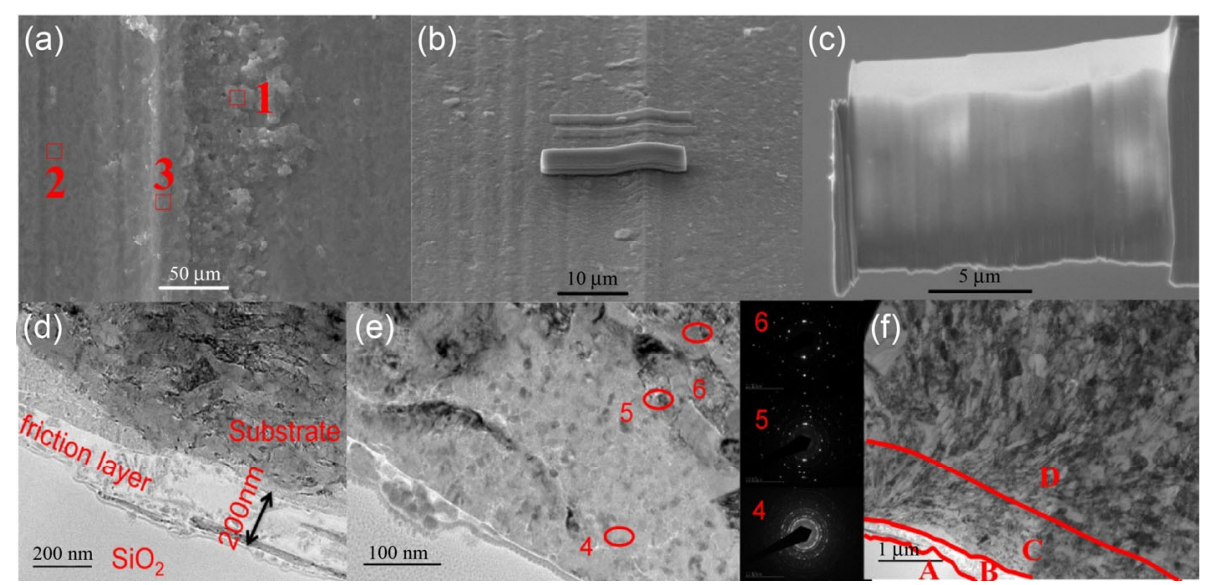

Fig. 23 SEM and TEM images of worn surfaces on disks lubricated with BP-16C at $75 \mathrm{mg} / \mathrm{L}$ : (a) SEM images of worn surfaces on disk; (b) the sampling place of samples using FIB; (c) TEM specimens by preparing in FIB; (d) TEM images of worn surfaces; (e) TEM images of substrate and the selected area electron diffraction (SAED) images; (f) TEM images of frictional layer.

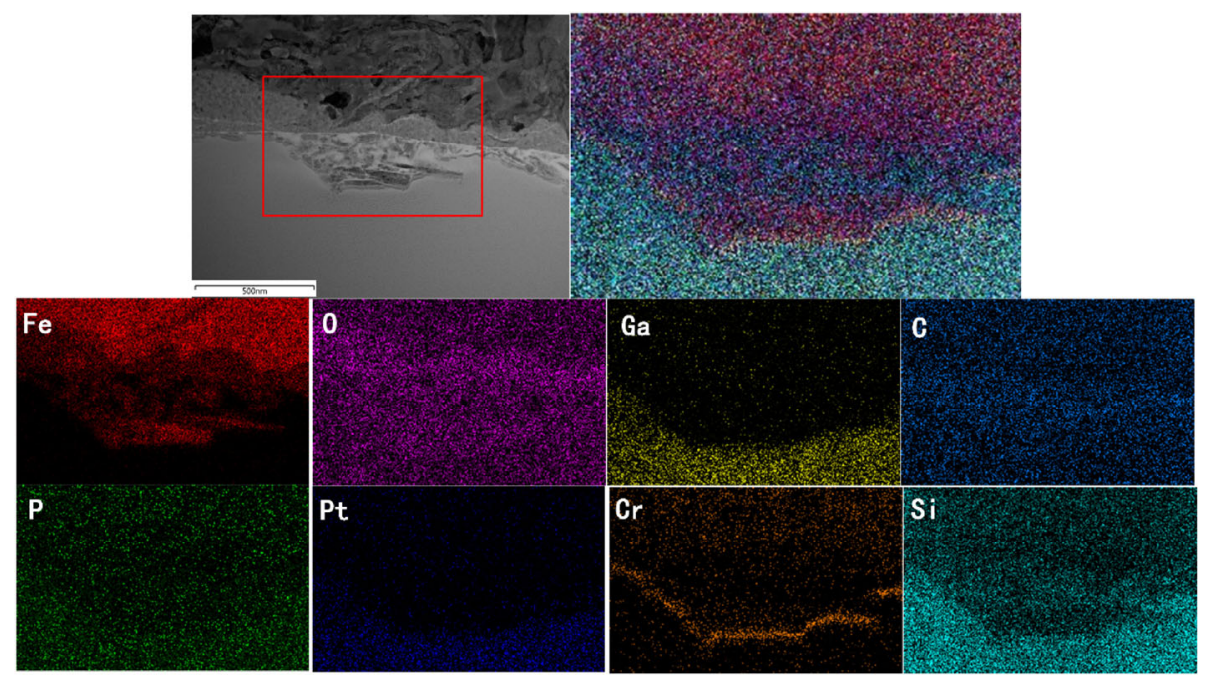

Fig. 24 The distribution of the chemical elements in frictional layer at worn surfaces of disks.

distribution of the chemical elements in the frictional layer of the substrate (disks). It can be observed from the red box that the rim of the friction layer is composed of $\mathrm{Cr}, \mathrm{Si}$ and $\mathrm{O}$. The protective coating protects the friction layer from damage during the FIB processing. The friction layer consisted of $\mathrm{Fe}, \mathrm{O}, \mathrm{C}$ and $\mathrm{P}$. Phosphorus was evenly distributed in the friction layer, and this result is different fromthe previous friction layeron the ball surface. The black nanosheets in the friction layer were composed of high-concentration $\mathrm{Fe}$ and $\mathrm{O}$, whereas the white area was composed of high-concentration $\mathrm{O}$ and $\mathrm{C}$. These results indicate that the friction layer was mainly composed of iron oxide, phosphide, and oxides of carbon.
In order to understand the lubrication mechanism of BP-16C, wear particles were collected on the wear scar of the steel ball after the friction test and they were investigated using TEM and HRTEM, as shown in Fig. 25. A variety of debris with different shapes and sizes can be observed and they are flat and elongated particles with a typical length of $20 \mathrm{~nm}$. The Debye-Scherrer electron diffraction pattern obtained for this particle presents several continuous rings centered at 2.127, 3.58, 3.892, 4.073, and $4.558 \mathrm{~nm}^{-1}$ (Fig. 25(b)). Inter-reticular distances calculated for these new rings (0.4701, 0.2793, 0.2569, 0.2455 and $0.2194 \mathrm{~nm}$, respectively) fit well with the distances in the $\mathrm{Fe}_{2} \mathrm{O}_{3}$ structure (orthorhombic iron oxide $\gamma-\mathrm{Fe}_{2} \mathrm{O}_{3}$, JCPDS data 


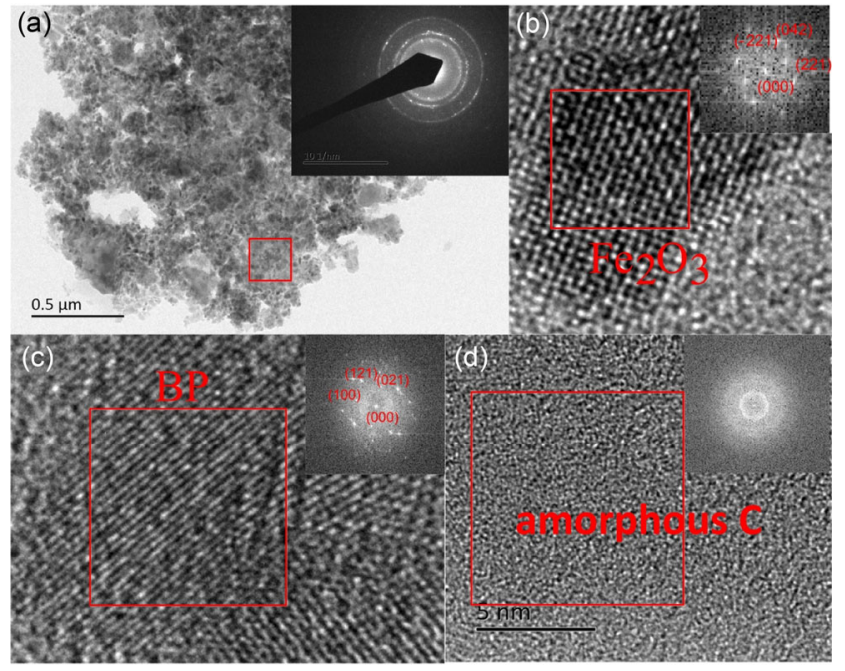

Fig. 25 TEM and HRTEM images of the wear debris.

$52-1499$ with cell $=0.5095 \mathrm{~nm} \times 0.8789 \mathrm{~nm} \times 0.9437 \mathrm{~nm})$ : $0.4718 \mathrm{~nm}$ for $\{002\}, 0.2798 \mathrm{~nm}$ for $\{311\}, 0.2560 \mathrm{~nm}$ for $\{113\}, 0.2452 \mathrm{~nm}$ for $\{131\}$ and $0.2197 \mathrm{~nm}$ for $\{040\}$. The BP nanosheets and amorphous carbon were also discovered in this microstructure using HRTEM and FFT pattern. The chemical composition of the debris is shown in Fig. 26. The chemical analysis shows that the debris mainly contained four elements: $\mathrm{Fe}, \mathrm{O}, \mathrm{P}$ and $\mathrm{C}$. The size of the $\mathrm{BP}$ nanosheets was varied. If the larger BP nanosheets could not enter the contact area, the distribution of the chemical elements in Fig. 27 explains the lubrication mechanism of BP-16C. Very small BP nanosheets (approximately $20 \mathrm{~nm}$ ) are shown in this figure and evenly distributed in the wear debris. This result indicates that the BP nanosheets entered into the contacted area, and reacted with the hexadecane and steel substrate.

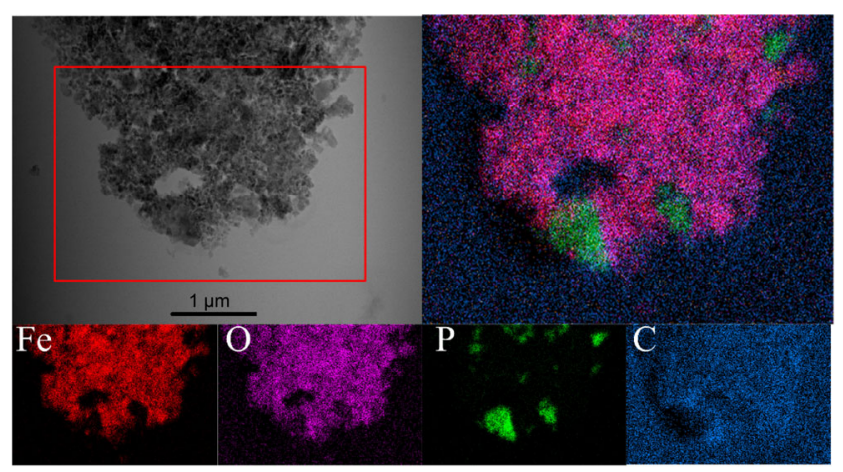

Fig. 26 The distribution of the chemical elements in wear debris.

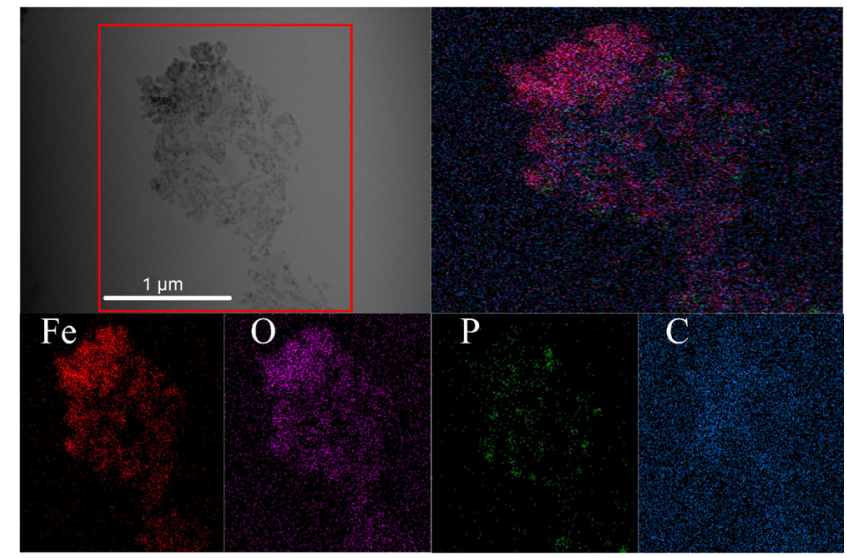

Fig. 27 The distribution of the chemical elements in wear debris.

In order to further explain the lubrication mechanism, Raman and XPS analysis were performed, as shown in Fig. 28. In the Raman spectrums (Fig. 28(a)), it can be seen that as the concentration of $\mathrm{BP}$ in hexadecane decreased, the strength of the characteristic peak also decreased. For the wear scar of the disk lubricated with the base oil, the typical peaks were detected at 656, 497, 403, 218 and $288 \mathrm{~cm}^{-1}$. Previous studies [75, 76] used Raman spectra for corrosion investigation of stainless steel and showed the characteristic peaks of iron oxides at $\sim 700 \mathrm{~cm}^{-1}$ and below. Maslar et al. [77] investigated the in-situ Raman spectroscopic of aqueous iron corrosion at elevated temperatures and pressures, and found that the characteristic peak at $656 \mathrm{~cm}^{-1}$ was detected, $497 \mathrm{~cm}^{-1}$ was the mixture of $\alpha$ and $\gamma-\mathrm{Fe}_{2} \mathrm{O}_{3}$, and the characteristic peaks among 403, 288 and 220 are all the $\alpha-\mathrm{Fe}_{2} \mathrm{O}_{3}$. Thus, the Raman signatures in our research of the wear tracks with BP-16C show the signal for iron oxides (below $700 \mathrm{~cm}^{-1}$ ). The peak of 1,316 $\mathrm{cm}^{-1}$ presented C-C stretch skeletal vibrations [78], that's means a protective film may have been formed by hexadecane in friction process. This lubricating film also reduced the friction and wear. These findings indicate that the deposition, self-repair, and formation of the chemical reaction film may be the main causes of the friction-reduction and anti-wear properties of BP-16C.

XPS analysis was performed to explore the lubricating mechanism of this lubrication additive. Figs. 28(b)-28(f) illustrate the full spectrum and curvefitted XPS spectra of C1s, Fe2p, O1s, and P2p on the wear scar of the disk lubricated with $10 \mathrm{mg} / \mathrm{L}$ 

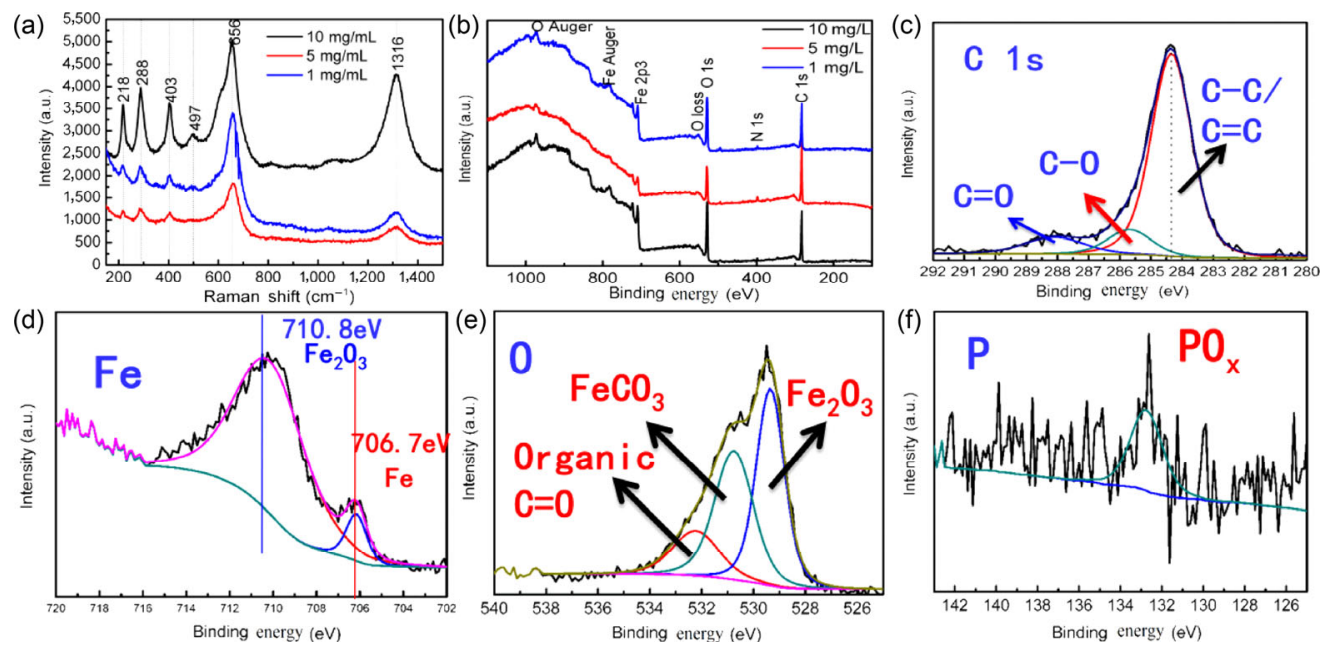

Fig. 28 Raman and XPS analysis of the worn surfaces on steel disks lubricated with the various concentration of BP-16C: (a) Raman of the worn surfaces; (b) XPS of the worn surfaces; (c) C, (d) Fe, (e) O, and (f) P obtained from the worn surfaces at the concentration of $1 \mathrm{mg} / \mathrm{L}$.

BP-dispersed oil. The Fe2p peak around $710.8 \mathrm{eV}$ is attributed to iron oxide and iron phosphate [79]. The Fe2p peaks around $706.7 \mathrm{eV}$ is attributed to the iron substrate. P2p peaks at $132.4 \mathrm{eV}$ is identified as $\mathrm{PO}_{4}^{3-}$. The N1s peak around $398.1 \mathrm{eV}$ is attributed to organic amine that comes from oleylamine using as the modifiers of dispersion. The Fe2p3/2 at $710.8 \mathrm{eV}$ and the O1s peak at $529.1 \mathrm{eV}$ both fall in the binding energy ranges covered by $\mathrm{Fe}_{2} \mathrm{O}_{3}$ [80]. Iron carbonate may still be present, but the $\mathrm{Fe} 2 \mathrm{p}$ peaks associated with $\mathrm{Fe}_{2} \mathrm{O}_{3}$ completely cover the traces. The indicator that $\mathrm{FeCO}_{3}$ was initially present is the small O1s peak at $530.9 \mathrm{eV}$ and $\mathrm{C} 1 \mathrm{~s}$ peak at $288.1 \mathrm{eV}$. In the carbon region the peak at $285.8 \mathrm{eV}$ attributed to the $\mathrm{C}-\mathrm{O}$ bond and the peak at $284.4 \mathrm{eV}$ attributed to the $\mathrm{C}-\mathrm{C} / \mathrm{C}=\mathrm{C}$ bond were identified. This was reported to be from the tribochemical reaction of hexadecane [81, 82]. Therefore, it can be concluded that a tribofilm made of BP and iron oxides was formed on the wear scar. These oxides play an important role in the friction-reducing ability of BP-16C.

The novel kind of oil-soluble BP nanosheets were prepared by phase transfer method. The tribology properties were evaluated by SRV-4 with ball-on-disc model. Schematic diagram of lubrication mechanism of BP nanosheets as lubrication additive was presented in Fig. 29. The aforementioned experimental results revealed that BP-16C has excellent lubrication properties under boundary lubrication. The lubrication mechanism with BP nanosheets as lubrication additives was concluded as follows. Firstly, when the micro peaks are moving each other, the ultrathin BP nanosheets will enter the contact area instead of being pushed away. When the contact pressure was applied to the touched micro peaks, because of the interlaminar shear between the few-layer the ultrathin BP nanosheets, they will not contact with each other directly. Secondly, shearing force in the hexadecane lubricant was another important impact factors. Finally, the plastic deformation of substrate was benefited for wear resistance. This study demonstrates that very low concentration of nicely dispersed BP nanosheets can generate the BP tribological thin film, which could

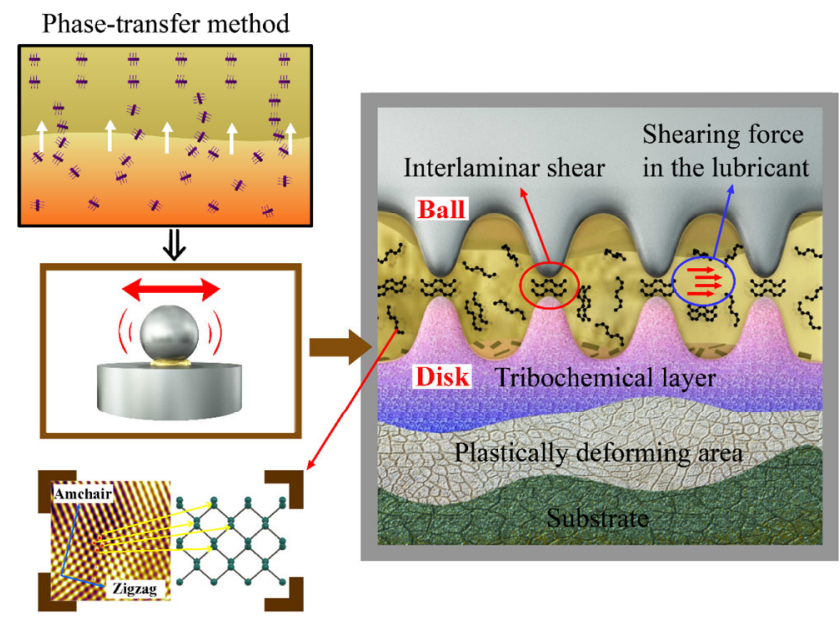

Fig. 29 Schematic diagram of lubrication mechanism of black phosphorus as lubrication additive. 
efficiently reduce the friction and protect the sliding interfaces. This promises the potential of BP-based lubricants for conserving energy by lowering the friction and reduction of material losses (wearpreventive property) for tribological applications.

\section{Friction properties of BP/PVDF composites materials}

\subsection{Preparation of BP/PVDF composites}

PVDF powders were purchased from J\&K Scientific Ltd., and BP powders were prepared using the HEMM method for $48 \mathrm{~h}$. The weight fractions of BP in the composites were $0,1 \%, 5 \%, 10 \%, 15 \%$ and $20 \%$. The $\mathrm{BP} / \mathrm{PVDF}$ composites were prepared according to the following steps (Fig. 30). First, a certain weight of BP and PVDF powders were weighed according to the target compositions, and subsequently wet ball-milled in anhydrous alcohol under argon atmosphere for $2 \mathrm{~h}$. The milling time and speed were $2 \mathrm{~h}$ and $300 \mathrm{rpm}$, respectively. After being mixed and dried, the mixtures were sintered via spark plasma sintering (SPS) at $250{ }^{\circ} \mathrm{C}$ under a pressure of $10 \mathrm{MPa}$ for $10 \mathrm{~min}$ in pure $\mathrm{Ar}$ atmosphere protection. A cylindrical graphite mold with an inner diameter of $20 \mathrm{~mm}$ was used.

\subsection{Characterization and friction properties of BP/PVDF composites}

The phase analysis of BP/PVDF composites was characterized using $\mathrm{XRD}$ with $\mathrm{Cu}-\mathrm{K} \alpha$ radiation within the $2 \theta$ range of $10-90^{\circ}$, and the molecular structure of the composites was analyzed using Raman spectra. The wavelength of laser light was $532 \mathrm{~nm}$, and the range of measured Raman shift was $100-1,100 \mathrm{~cm}^{-1}$.

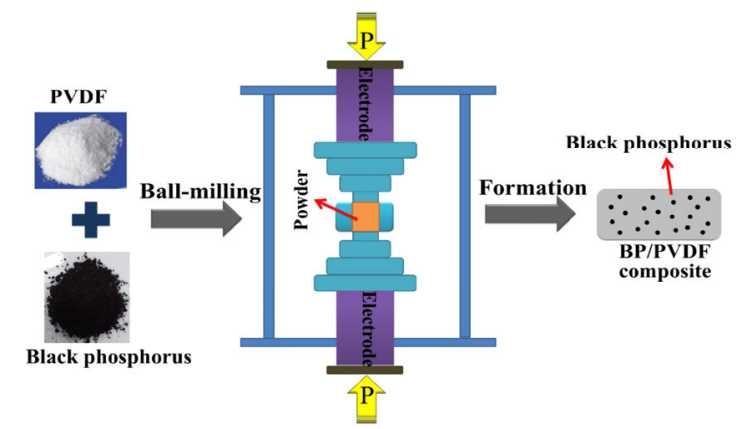

Fig. 30 Schematic diagram of preparation process of BP/PVDF composites.
The morphologies of BP/PVDF composites were characterized using SEM. The friction test was conducted on a universal micro-tribotester (UMT-5), and the reciprocating speed was $0.03 \mathrm{~m} / \mathrm{s}$. Stainless steel balls of diameter $4.4 \mathrm{~mm}$ were used. The sliding experiments were performed over a period of $30 \mathrm{~min}$ and at the applied loads of 1, 3 and $5 \mathrm{~N}$.

\subsection{Results and discussion}

The XRD patterns of BP, PVDF and BP/PVDF composites with different $\mathrm{BP}$ contents are shown in Fig. 31(a). The XRD peaks at $2 \theta$ of $16.8^{\circ}, 26.6^{\circ}, 34.9^{\circ}$ and $55.5^{\circ}$ correspond to the (020), (021), (111) and (132) crystal planes of BP, respectively. The XRD peaks at $2 \theta$ of $18.4^{\circ}, 19.9^{\circ}$ and $26.4^{\circ}$ correspond to the (020), (110) and (021) crystal planes of PVDF, respectively. The XRD patterns of BP/PVDF composites prepared by SPS are also presented in Fig. 31(a). The three strongest peaks of BP are marked in the graph, corresponding to the (111), (112), and (132) crystal planes. All the patterns of BP/PVDF composites show (111) peaks similar to that of pure BP, and the peak intensity increases with the increase in BP content. However, the (112) and (132) peaks cannot be clearly observed in the composites. The Raman spectroscopies of BP, PVDF, and BP/PVDF composites with different $\mathrm{BP}$ contents are presented in Fig. 31(b). In BP, the peaks of $359 \mathrm{~cm}^{-1}, 436 \mathrm{~cm}^{-1}$ and $462 \mathrm{~cm}^{-1}$, corresponding to $\mathrm{A}_{\mathrm{g}}^{1}, \mathrm{~B}_{2 \mathrm{~g}}$ and $\mathrm{A}_{\mathrm{g}}^{2}$ phonon modes, respectively, can be observed. The strongest peak of PVDF was observed at $796 \mathrm{~cm}^{-1}$. The Raman spectra of BP/PVDF composites with different BP contents are also shown in Fig. 31(b). In all the composites, the characteristic peaks of BP were also presented, and moreover, as the content of BP in the composites increased,
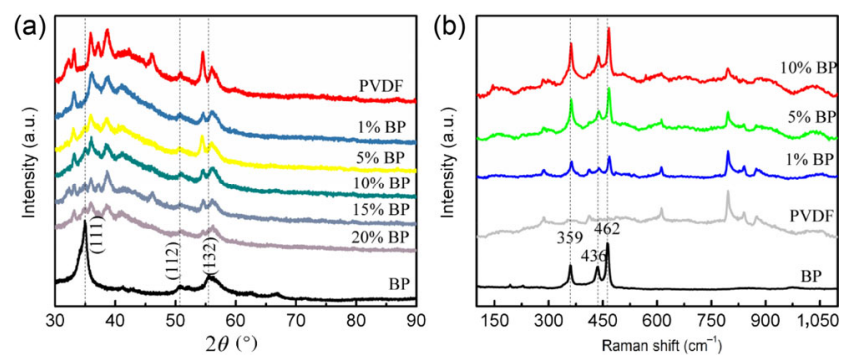

Fig. 31 (a) XRD patterns of BP, PVDF and BP/PVDF composites with different BP contents; (b) Raman spectra of BP, PVDF, and $\mathrm{BP} / \mathrm{PVDF}$ composites with different BP contents. 
the intensities of peaks increased correspondingly. Figure 32 shows the SEM images of the composites with BP contents of 15 wt.\% and 20 wt.\%. The BP particles were properly dispersed in the PVDF matrix, and the size of the BP particles increased with the increase in the content of BP. However, the BP particles in the composites tended to aggregate with the increase in content of BP.

The mean COF value of BP/PVDF composites as a function of BP content and applied load are shown in Fig. 33. Under a constant applied load, the COF increased with the increase in the BP content, and reached the highest value when the $\mathrm{BP}$ content was approximately $10 \mathrm{wt} . \%$. However, the COF value decreased rapidly as the content of BP was increased further. It is shown in Fig. 34 that, when the BP content in the BP/PVDF composite is lower than $10 \mathrm{wt} . \%$, the addition of BP filler destroys the semicrystalline structure of PVDF, resulting in the decrease of the yield strength, and correspondingly the COF of the composite decreases. In the case of the composite with BP content larger than $10 \mathrm{wt} . \%$, the BP filler plays a more important role in the lubricating process. In order to demonstrate the results of this analysis, the wear scar region was analyzed using the Raman spectrometer. As shown in Fig. 35, the Raman spectra

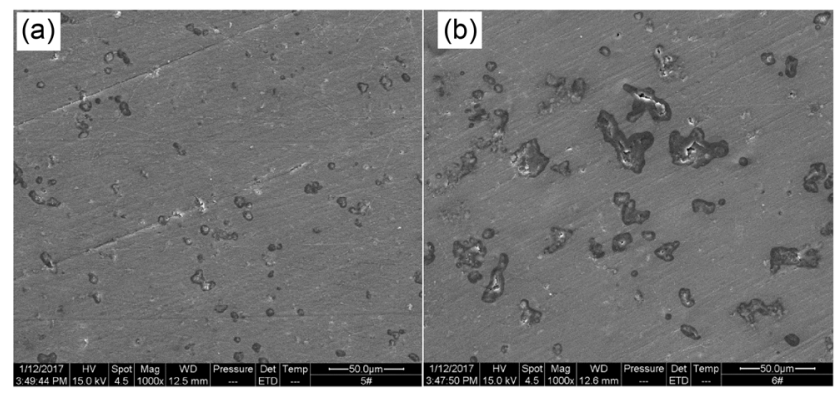

Fig. 32 SEM micrograph of composites with BP content of (a) $15 \mathrm{wt} \%$; (b) $20 \mathrm{wt} \%$. in the wear scar and on the surface of the composite show similar peaks at $362 \mathrm{~cm}^{-1}, 432 \mathrm{~cm}^{-1}$ and $461 \mathrm{~cm}^{-1}$ owing to the BP filler, and at $794 \mathrm{~cm}^{-1}$ owing to the PVDF. This indicates that chemical reactions did not occur during the sliding process. Therefore, it can be inferred that the improvement of friction properties is ascribed to the increasing amount of BP filler and its lubricating properties.

However, the COFs of BP/PVDF composites with certain BP contents decreased with the increase in applied load. Considering the composite with $10 \mathrm{wt} . \%$ $\mathrm{BP}$ content as an example, the COF value decreased from 0.32 to 0.24 when the load increased from 1 to $5 \mathrm{~N}$. A possible explanation is that the friction surface experienced not only plastic deformation but also elastic deformation [5]. In this case, the COF is inversely proportional to applied load, and shows a decreasing trend with the increase in load. Figure 36 shows the wear rate of BP/PVDF composites at different applied loads as a function of BP content. It can be observed that, under a certain applied load, the wear rate decreases rapidly with the increase in the content of BP filler. For instance, when the load was $5 \mathrm{~N}$,

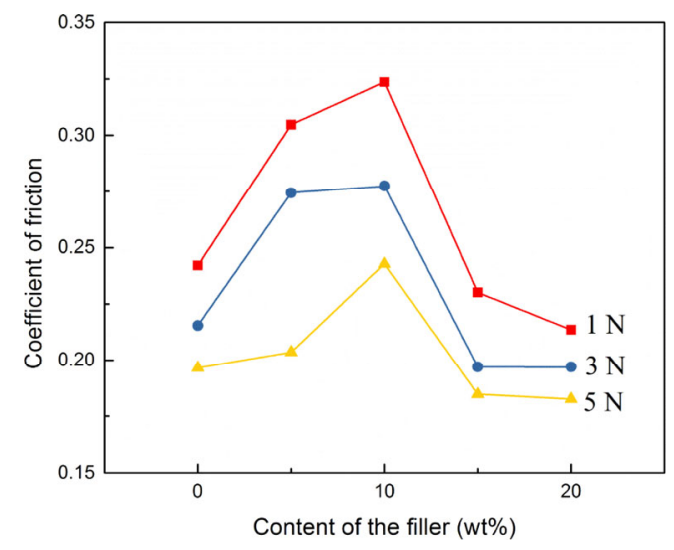

Fig. 33 The mean friction coefficient as a function of BP content under different loads.
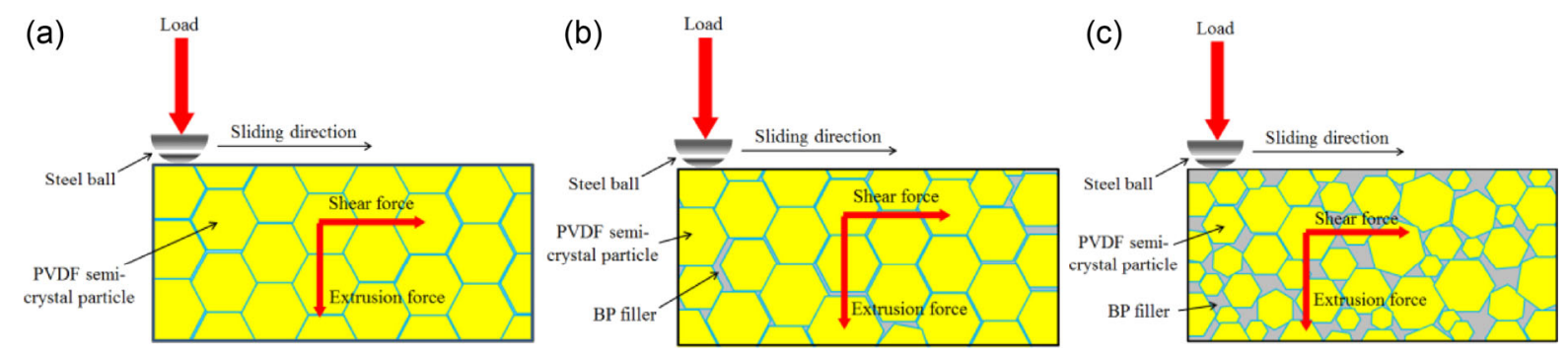

Fig. 34 Schematic diagram of the friction process of composites with different BP contents: (a) neat PVDF; (b) low BP content; (c) high BP content. 


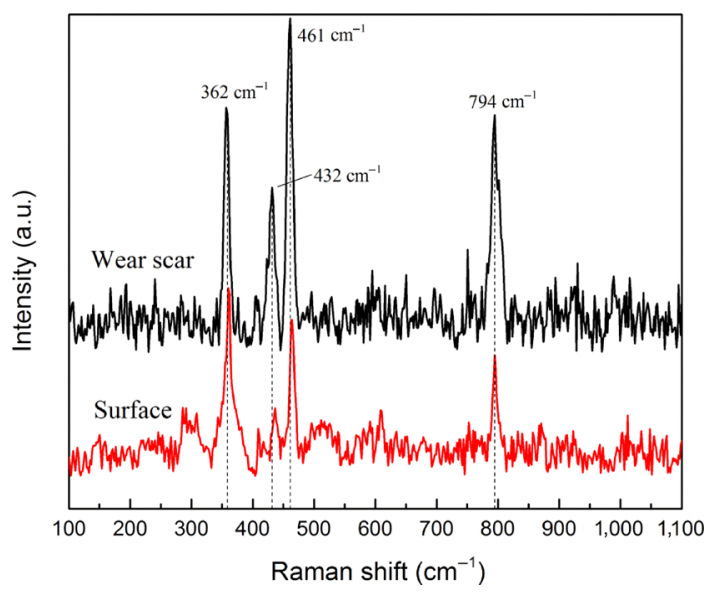

Fig. 35 Raman spectra of wear scar and surface of composites with BP content of 15 wt $\%$.

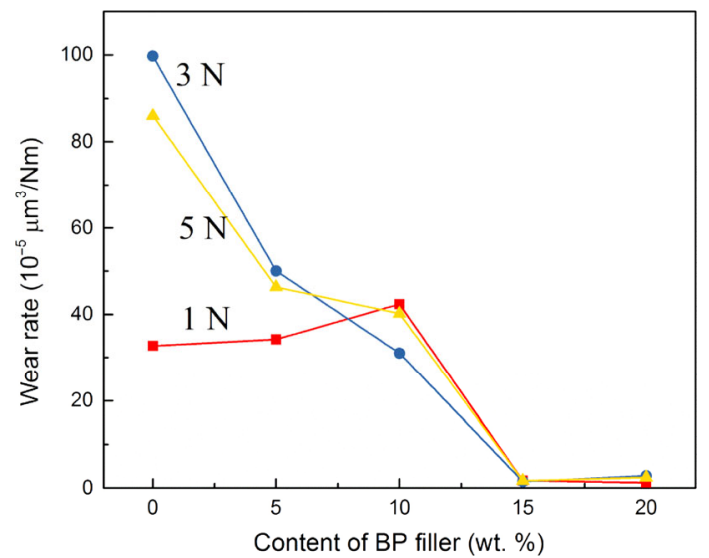

Fig. 36 Wear rate of BP/PVDF composites at different applied loads.

the wear rate reduced from $8.6 \times 10^{-4} \mathrm{~mm}^{3} /(\mathrm{N} \cdot \mathrm{m})$ to $2.5 \times 10^{-5} \mathrm{~mm}^{3} /(\mathrm{N} \cdot \mathrm{m})$ with the BP filler increasing from 0 to $20 \mathrm{wt} . \%$. This indicates that the addition of BP filler is beneficial to the reduction in the wear loss of the composites.

\section{Conclusion and outlook}

In this work, the synthesis of BP nano-powders using the HEEM technique, modification of BP nanosheets as water-based and oil-based lubricant additives and the tribological characteristics of few-layer BP nanosheets have been systematically studied. Moreover, the tribological characteristics of $\mathrm{MoS}_{2}$ and GO nanosheets as oil-based lubricant additives were also investigated for comparison. Furthermore, the tribological properties of polymeric composites with $\mathrm{BP}$ as the filler were also investigated. It was observed that, owing to the oxidation process in ambient environment, the Raman intensities at different air exposure times decreased initially and subsequently remained steady owing to the formation of oxidation layers, resulting in the increase in friction in the microscale. For the use of BP nanosheets as oil-based lubricant additives, the BP dispersions showed similar lubrication properties at low loads, whereas the BP dispersions exhibited outstanding resistance to extreme pressure and bearing capacity at high loads. For BP/PVDF composites, when the BP content was larger than $10 \%$, the improvement of friction properties was more apparent, and the addition of BP could enhance the wear resistance of $\mathrm{BP} / \mathrm{PVDF}$ composites.

In spite of the current progresses of the tribological properties of BP, several challenges still remain. First, the methods for cost-effective mass production of $\mathrm{BP}$ nanomaterials are still required. Second, while the small lateral size $\left(<1 \mu \mathrm{m}^{2}\right)$ of most nanosheet dispersions is sufficient for many technologies in tribological applications, larger flake size is more desirable for lubrication additives applications. Third, further work on the ultra-low friction properties of BP-based lubricant or composite is necessary.

\section{Acknowledgements}

The authors would like to acknowledge Xudong Chen, Ziyi Cui, Jinjin Li and Dan Guo for some of experiments present in the manuscript and their helpful discussions. The authors also would like to acknowledge the support of the National Natural Science Foundation of China (Grant Nos. 51527901, 51335005, 51475256, and 51605249).

Open Access: The articles published in this journal are distributed under the terms of the Creative Commons Attribution 4.0 International License (http:// creativecommons.org/licenses/by/4.0/), which permits unrestricted use, distribution, and reproduction in any medium, provided you give appropriate credit to the original author(s) and the source, provide a link to the Creative Commons license, and indicate if changes were made. 


\section{References}

[1] Dasic P. International standardization and organizations in the field of tribology. Ind Lubr Tribol 55(6): 287-291 (2003)

[2] Jost H P. Tribology micro \& macroeconomics: A road to economic savings. Tribol Lubr Technol 61(10): 18-22 (2005)

[3] Iliuc I. Plenary lecture V: EU objective of $120 \mathrm{~g} \mathrm{CO}_{2} / \mathrm{km}$ emission for new cars a challenge for tribology. In Proceedings of the 9th WSEAS International Conference on Mathematics \& Computers in Biology \& Chemistry, Bucharest, Romania, 2008: 15.

[4] Xia Y Q, Xu X C, Feng X, Chen G X. Leaf-surface wax of desert plants as a potential lubricant additive. Friction 3(3): 208-213 (2015)

[5] $\mathrm{Xu} \mathrm{J,} \mathrm{Li} \mathrm{J} \mathrm{J.} \mathrm{New} \mathrm{achievements} \mathrm{in} \mathrm{superlubricity} \mathrm{from}$ international workshop on superlubricity: Fundamental and applications. Friction 3(4): 344-351 (2015)

[6] Li J J, Zhang C H, Luo J B. Superlubricity behavior with phosphoric acid-water network induced by rubbing. Langmuir 27(15): 9413-9417 (2011)

[7] Novoselov K S, Jiang D, Schedin F, Booth T J, Khotkevich V V, Morozov S V, Geim A K. Two-dimensional atomic crystals. Proc Natl Acad Sci USA 102(30): 10451-10453 (2005)

[8] Filleter T, Bennewitz R. Structural and frictional properties of graphene films on $\mathrm{SiC}(0001)$ studied by atomic force microscopy. Phys Rev B 81(15): 155412 (2010)

[9] Dienwiebel M, Verhoeven G S, Pradeep N, Frenken J W M, Heimberg J A, Zandbergen H W. Superlubricity of graphite. Phys Rev Lett 92(12): 126101 (2004)

[10] Chu T, Ilatikhameneh H, Klimeck G, Rahman R, Chen Z H. Electrically tunable bandgaps in bilayer $\mathrm{MoS}_{2}$. Nano Lett 15(12): 8000-8007 (2015)

[11] Kumari S, SharamaO P, Gusain R, Mungse H P, Kukrety A, Kumar N, Sugimura H, Khatri O P. Alkyl-chain-grafted hexagonal boron nitride nanoplatelets as oil-dispersible additives for friction and wear reduction. ACS Appl Mater Interfaces 7(6): 3708-3716 (2015)

[12] Erdemir A, Eryilmaz O. Achieving superlubricity in DLC films by controlling bulk, surface, and tribochemistry. Friction 2(2): 140-155 (2014)

[13] Ataca C, Şahin H, Aktürk E, Ciraci S. Mechanical and electronic properties of $\mathrm{MoS}_{2}$ nanoribbons and their defects. J Phys Chem C 115(10): 3934-3941 (2011)

[14] Berman D, Erdemir A, Sumant A V. Few layer graphene to reduce wear and friction on sliding steel surfaces. Carbon 54: 454-459(2013)

[15] Liu D E, Xie G X, Guo D, Cui Z Y, Si L N, Wan C L, Zou W, Luo J B. Tunable lubricity of aliphatic ammonium graphite intercalation compounds at the micro/nanoscale. Carbon 115: 574-583 (2017)
[16] Guo W L, Yin J, Qiu H, Guo Y F, Wu H R, Xue M M. Friction of low-dimensional nanomaterial systems. Friction 2(3): 209-225 (2014)

[17] Zhang W, Zhou M, Zhu H W, Tian Y, Wang K L, Wei J Q, Ji F, Li X, Li Z, Zhang P, et al. Tribological properties of oleic acid-modified graphene as lubricant oil additives. J Phys DAppl Phys 44(20): 205303 (2011).

[18] Wang H D, Liu Y H, Liu W R, Wang R, Wen J G, Sheng H P, Feng J f, Erdemir A, Luo J B.Tribological Behavior of NiAl-Layered Double Hydroxide Nanoplatelets as Oil-Based Lubricant Additives. ACS Appl. Mater. Interfaces 9 (36): 30891-30899 (2017).

[19] Kogovšek J, Kalin M. Various $\mathrm{MoS}_{2^{-}}, \mathrm{WS}_{2^{-}}$and C-based micro-and nanoparticles in boundary lubrication. Tribol Lett 53(3): 585-597 (2014)

[20] Chen Z, Liu X W, Liu Y H, GunselS, Luo J B. Ultrathin $\mathrm{MoS}_{2}$ nanosheets with superior extreme pressure property as boundary lubricants. Sci Rep 5: 12869 (2015)

[21] Xie G X, Forslund M, Pan J S. Direct electrochemical synthesis of reduced graphene oxide (rGO)/copper composite films and their electrical/electroactive properties. ACS Appl Mater Interfaces 6(10): 7444-7455 (2014)

[22] Belmonte M, Ramírez C, González-Julián J, Schneider J, Miranzo P, Osendi M I. The beneficial effect of graphene nanofillers on the tribological performance of ceramics. Carbon 61: 431-435 (2013)

[23] Sorrentino A, Altavilla C, Merola M, Senatore A, Ciambelli P, Iannace S. Nanosheets of $\mathrm{MoS}_{2}$-oleylamine as hybrid filler for self-lubricating polymer composites: Thermal, tribological, and mechanical properties. Polym Compos 36(6): 1124-1134 (2015)

[24] Ling X, Wang H, Huang S X, Xia F N, Dresselhaus M S. The renaissance of black phosphorus. Proc Natl Acad Sci USA 112(15): 4523-4530 (2015)

[25] Tao J, Shen W F, Wu S, Liu L, Feng Z H, Wang C, Hu C G, Yao P, Zhang H, Pang W, et al. Mechanical and electrical anisotropy of few-layer black phosphorus. ACS Nano 9(11): 11362-11370 (2015)

[26] Chen H, Huang P, Guo D, Xie G X. Anisotropic mechanical properties of black phosphorus nanoribbons. J Phys Chem C 120(51): 29491-29497 (2016)

[27] Liu H, Du Y C, Deng Y X, Ye P D. Semiconducting black phosphorus: Synthesis, transport properties and electronic applications. Chem Soc Rev 44(9): 2732-2743 (2015)

[28] Lei W Y, Liu G, Zhang J, Liu M H. Black phosphorus nanostructures: Recent advances in hybridization, doping and functionalization. Chem Soc Rev 46(12): 3492-3509 (2017)

[29] Lee T H, Kim S Y, Jang H W. Black phosphorus: Critical review and potential for water splitting photocatalyst. Nanomaterials 6(12): 194 (2016) 
[30] Castellanos-Gomez A. Black phosphorus: Narrow gap, wide applications. J Phys Chem Lett 6(21): 4280-4291 (2015)

[31] Du Y L, Ouyang C Y, Shi S Q, Lei M S. Ab initio studies on atomic and electronic structures of black phosphorus. $J$ Appl Phys 107(9): 093718 (2010)

[32] Kim H. Effect of van der Waals interaction on the structural and cohesive properties of black phosphorus. J Korean Phys Soc 64(4): 547-553 (2014)

[33] Wang Z H, Feng P X L. Design of black phosphorus 2D nanomechanical resonators by exploiting the intrinsic mechanical anisotropy. 2D Mater 2(2): 021001 (2015)

[34] Brent J R, Savjani N, Lewis E A, Haigh S J, Lewis D J, O'Brien P. Production of few-layer phosphorene by liquid exfoliation of black phosphorus. Chem Commun 50(87): 13338-13341 (2014)

[35] Bridgman P W. Two new modifications of phosphorus. $J$ Am Chem Soc 36(7): 1344-1363 (1914)

[36] Maruyama Y, Suzuki S, Kobayashi K, Tanuma S. Synthesis and some properties of black phosphorus single crystals. Phys BC 105(1-3): 99-102 (1981)

[37] Shirotani I. Growth of large single crystals of black phosphorus at high pressures and temperatures, and its electrical properties. Mol Cryst Liq Cryst 86(1): 203-211 (1982)

[38] Endo S, Akahama Y, Terada S, Narita S. Growth of large single crystals of black phosphorus under high pressure. Jpn J Appl Phys 21(8): L482-L484 (1982)

[39] Park C M, Sohn H J. Black phosphorus and its composite for lithium rechargeable batteries. Adv Mater 19(18): 24652468 (2007)

[40] Krebs H, Schultze-Gebhardt F. Über die Struktur und Eigenschaften der Halbmetalle. VII. Neubestimmung der Struktur des glasigen Selens nach verbesserten röntgenographischen Methoden. Acta Crystallogr 8(7): 412-419 (1955)

[41] Nilges T, Kersting M, Pfeifer T. A fast low-pressure transport route to large black phosphorus single crystals. J Solid State Chem 181(8): 1707-1711 (2008)

[42] Liu H, Neal A T, Zhu Z, Luo Z, Xu X F, Tománek D, Ye P D. Phosphorene: An unexplored 2D semiconductor with a high hole mobility. ACS Nano 8(4): 4033-4041 (2014)

[43] Lu W L, Nan H Y, Hong J H, Chen Y M, Zhu C, Liang Z, Ma X Y, Ni Z H, Jin C H, Zhang Z. Plasma-assisted fabrication of monolayer phosphorene and its Raman characterization. Nano Res 7(6): 853-859 (2014)

[44] YasaeiP, KumarB, ForoozanT, Wang C H, AsadiM, TuschelD, IndacocheaJ E, KlieR F, Salehi-KhojinA. High-quality black phosphorus atomic layers by liquid-phase exfoliation. $A d v$ Mater 27(11): 1887-1892 (2015)

[45] Bagheri S, Mansouri N, Aghaie E. Phosphorene: A new competitor for graphene. Int J Hydrogen Energy 41(7): 4085-4095 (2016)
[46] Rodin A S, Carvalho A, Castro Neto A H. Strain-induced gap modification in black phosphorus. Phys Rev Lett 112(17): 176801 (2014)

[47] Asahina H, Morita A. Band structure and optical properties of black phosphorus. J Phys C Solid State Phys 17(11): 1839-1852 (1984)

[48] Liu B L, Köpf M, Abbas A N, Wang X M, Guo Q S, Jia Y C, Xia F N, Weihrich R, Bachhuber F, Pielnhofer F, Wang H, Dhall R, Cronin S B, Ge M Y, Fang X, Nilges T, Zhou C W. Black arsenic-phosphorus: Layered anisotropic infrared semiconductors with highly tunable compositions and properties. Adv Mater 27: 4423-4429 (2015).

[49] Wang Q H, Kalantar-Zadeh K, Kis A, Coleman J N, Strano M S. Electronics and optoelectronics of two-dimensional transition metal dichalcogenides. Nat Nanotechnol 7(11): 699-712 (2012)

[50] Lv R T, Robinson J A, Schaak R E, Sun D, Sun Y F, Mallouk T E, Terrones M. Transition metal dichalcogenides and beyond: Synthesis, properties, and applications of singleand few-layer nanosheets. Acc Chem Res 48(1): 56-64 (2015)

[51] Xia F N, Wang H, Xiao D, Dubey M, Ramasubramaniam A. Two-dimensional material nanophotonics. Nat Photonics 8(12): 899-907 (2014)

[52] Gusmão R, Sofer Z, Pumera M. Black phosphorus rediscovered: From bulk material to monolayers. Angew Chem Int Ed Engl 56(28): 8052-8072 (2017)

[53] Jiang J W, Park H S. Mechanical properties of single-layer black phosphorus. J Phys D Appl Phys 47(38): 385304 (2014)

[54] Qin G Z, Yan Q B, Qin Z Z, Yue S Y, Hu M, Su G. Anisotropic intrinsic lattice thermal conductivity of phosphorene from first principles. Phys Chem Chem Phys17(7): 4854-4858 (2015)

[55] Zhang Y Y, Pei Q X, Jiang J W, Wei N, Zhang Y W. Thermal conductivities of single- and multi-layer phosphorene: A molecular dynamics study. Nanoscale 8(1): 483-491 (2016)

[56] Huang Y, Qiao J S, He K, Bliznakov S, Sutter E, Chen X J, Luo D, Meng F K, Su D, Decker J, et al. Interaction of black phosphorus with oxygen and water. Chem Mater 28(22) 8330-8339 (2016)

[57] O'Hare P A G, Lewis B M, Shirotani I. Thermodynamic stability of orthorhombic black phosphorus. Thermochim Acta 129(1): 57-62 (1988)

[58] Li L K, Yu Y J, Ye G J, Ge Q Q, Ou X D, Wu H, Feng D L, Chen X H, Zhang Y B. Black phosphorus field-effect transistors. Nat Nanotechnol 9(5): 372-377 (2014)

[59] Zhu W N, Yogeesh M N, Yang S X, H. Aldave S H, Kim J S, Sonde S, Tao L, Lu N S, Akinwande D. Flexible black phosphorus ambipolar transistors, circuits and AM demodulator. Nano Lett 15(3): 1883-1890 (2015) 
[60] Abbas A N, Liu B L, Chen L, Ma Y Q, Cong S, Aroonyadet N, Köpf M, Nilges T, Zhou C W. Black phosphorus gas sensors. ACS Nano 9(5): 5618-5624 (2015)

[61] Cho S Y, Lee Y, Koh H J, Jung H, Kim J S, Yoo H W, Kim J H, Jung H T. Superior chemical sensing performance of black phosphorus: Comparison with $\mathrm{MoS}_{2}$ and graphene. Adv Mater 28(32): 7020-7028 (2016)

[62] Miao J S, Cai L, Zhang S M, Nah J, Yeom J, Wang C. Air-stable humidity sensor using few-layer black phosphorus. ACS Appl Mater Interfaces 9(11): 10019-10026 (2017)

[63] Sun J, Lee H W, Pasta M, Yuan H T, Zheng G Y, Sun Y M, Li Y Z, Cui Y. A phosphorene-graphene hybrid material as a high-capacity anode for sodium-ion batteries. Nat Nanotechnol 10(11): 980-985 (2015)

[64] Shen Z R, Sun S T, Wang W J, Liu J W, Liu Z F, Yu J C. A black-red phosphorus heterostructure for efficient visiblelight-driven photocatalysis. J Mater Chem A 3(7): 3285-3288 (2015)

[65] Sa B S, Li Y L, Qi J S, Ahuja R, Sun Z M. Strain engineering for phosphorene: The potential application as a photocatalyst. J Phys Chem C 118(46): 26560-26568 (2014)

[66] Zhang X, Xie H M, Liu Z D, Tan C L, Luo Z M, Li H, Lin J D, Sun L Q, Chen W, Xu Z C, et al. Black phosphorus quantum dots. Angew Chem Int Ed Engl 54(12): 3653-3657 (2015)

[67] Moreno-Moreno M, Lopez-Polin G, Castellanos-Gomez A, Gomez-Navarro C, Gomez-Herrero J. Environmental effects in mechanical properties of few-layer black phosphorus. $2 D$ Mater 3(3): 031007 (2016)

[68] Jiang J W, Park H S. Mechanical properties of single-layer black phosphorus. J Phys D Appl Phys 47(38): 385304 (2014)

[69] Nagao M, Hayashi A, Tatsumisago M. All-solid-state lithium secondary batteries with high capacity using black phosphorus negative electrode. J Power Sources 196(16): 6902-6905 (2011)

[70] Sun C X, Wen L, Zeng J F, Wang Y, Sun Q, Deng L J, Zhao C J, Li Z. One-pot solventless preparation of PEGylated black phosphorus nanoparticles for photoacoustic imaging and photothermal therapy of cancer. Biomaterials 91: 81-89 (2016)

[71] Castellanos-Gomez A, Vicarelli L, Prada E, Island J O,

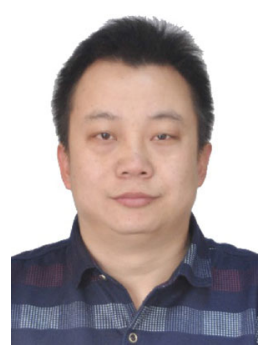

Wei WANG. He received the Ph.D. degree in Materials Processing Engineering from Northwestern Polytechnical University, Xi'an, China, in 2015. After a two-year post-doctoral work at State Key
Narasimha-Acharya K L, Blanter S I, Groenendijk D J, Buscema M, Steele G A, Alvarez J V, et al. Isolation and characterization of few-layer black phosphorus. 2D Mater 1(2): 025001 (2014)

[72] Island J O, Steele G A, van der Zant H S J, CastellanosGomez A. Environmental instability of few-layer black phosphorus. 2D Mater 2(1): 011002 (2015)

[73] Edmonds M T, Tadich A, Carvalho A, Ziletti A, O’Donnell K M, Koenig S P, Coker D F, Özyilmaz B, Castro Neto A H, Fuhrer M S. Creating a stable oxide at the surface of black phosphorus. ACS Appl Mater Interfaces 7(27): 14557-14562 (2015)

[74] Late D J. Liquid exfoliation of black phosphorus nanosheets and its application as humidity sensor. Microporous Mesoporous Mater 225: 494-503 (2016)

[75] Maslar J E, Hurst W S, Bowers Jr W J, Hendricks J H. In situ Raman spectroscopic investigation of stainless steel hydrothermal corrosion. Corrosion 58(9): 739-747 (2002)

[76] Oblonsky L J, Devine T M. A surface enhanced Raman spectroscopic study of the passive films formed in borate buffer on iron, nickel, chromium and stainless steel. Corros Sci 37(1): 17-41 (1995)

[77] Maslar J E, Hurst W S, Bowers W J, HendricksJ H, Aquino M I. In Situ Raman spectroscopic investigation of aqueous iron corrosion at elevated temperatures and pressures. $J$ Electrochem Soc 147(7): 2532-2542 (2000)

[78] $\mathrm{Hu} \mathrm{Z} \mathrm{S,} \mathrm{Hsu} \mathrm{S} \mathrm{M,} \mathrm{Wang} \mathrm{P} \mathrm{S.} \mathrm{Tribochemical} \mathrm{reaction} \mathrm{of}$ stearic acid on copper surface studied by surface enhanced Raman spectroscopy. Tribol Trans 35(3): 417-422 (1992)

[79] Ma H B, Li J, Chen H, Zuo G X, Yu Y, Ren T H, Zhao Y D. XPS and XANES characteristics of tribofilms and thermal films generated by two P- and/or S-containing additives in water-based lubricant. Tribol Int 42(6): 940-945 (2009)

[80] Heuer J K, Stubbins J F. An XPS characterization of $\mathrm{FeCO}_{3}$ films from $\mathrm{CO}_{2}$ corrosion. Corros Sci 41(7): 1231-1243 (1999)

[81] Kajdas C, Makowska M, Gradkowski M. Tribochemistry of $n$-hexadecane in different material systems. Lubr Sci 18(4): 255-263 (2006)

[82] Kajdas C, Makowska M, Gradkowski M. Influence of temperature on the tribochemical reactions of hexadecane. Lubr Sci 15(4): 329-340 (2003)

Laboratory of Tribology of Tsinghua University, he is currently an associate professor from Xi'an University of Architecture and Technology. Dr. Wang has published more than 10 papers indexed by SCI as the first author. His major research areas include lubrication in material processing and nano-lubrication. 


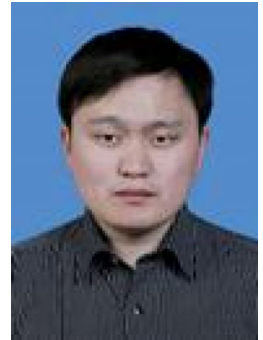

Guoxin XIE. He received his Ph.D. degree at Tsinghua University, China, in 2010, majoring in Mechanical Engineering. After that, he spent two years at State Key Laboratory of Tribology, Tsinghua University, China for postdoctoral research. From 2012 to 2014, he worked at Royal Institute of Technology, Sweden, for

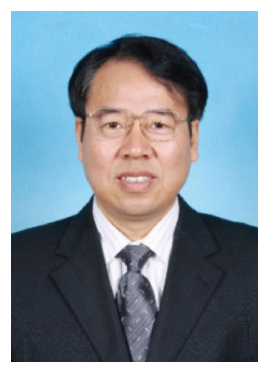

Jianbin LUO. He received his BEng degree from Northeastern University in 1982, and got his MEng degree from Xi'an University of Architecture and Technology in 1988. In 1994, he received his PhD degree from Tsinghua University and then joined the faculty of Tsinghua University. Prof. Jianbin Luo is an academician of the Chinese Academy of Sciences and a Yangtze another two years' post-doctoral research. Since 2014, he has worked at Tsinghua University as an associate Professor. His research interests include intelligent self-lubrication, electric contact lubrication, etc. He has published more than 50 referred papers in international journals. He won several important academic awards, such as Chinese Thousands of Young Talents, the Excellent Doctoral Dissertation Award of China, and Ragnar Holm Plaque from KTH, Sweden.

River Scholar Distinguished Professor of Tsinghua University, Beijing, China. He was awarded the STLE International Award (2013), the Chinese National Technology Progress Prize (2008), the Chinese National Natural Science Prize (2001), and the Chinese National Invention Prize (1996). Prof. Luo has been engaged in the research of thin film lubrication and tribology in nanomanufacturing. He was invited as a keynote or plenary speaker for 20 times on the international conferences. 